\title{
The origin, excitation, and evolution of subarcsecond outflows near T Tauri ${ }^{\star}$
}

\author{
M. Gustafsson ${ }^{1}$, L. E. Kristensen ${ }^{2}$, M. Kasper ${ }^{3}$, and T. M. Herbst ${ }^{1,4}$ \\ 1 Max Planck Institute for Astronomy, Königstuhl 17, 69117 Heidelberg, Germany \\ e-mail: [gustafsson; herbst] @mpia.de \\ 2 Leiden Observatory, Leiden University, PO Box 9513, 2300 RA Leiden, The Netherlands \\ e-mail: kristensen@strw. leidenuniv.nl \\ 3 European Southern Observatory, Karl-Schwarzschild-Str. 2, 85748 Garching, Germany \\ e-mail: mkasper@eso.org \\ ${ }^{4}$ Herzberg Institute of Astrophysics, National Research Council of Canada, Victoria, BC V9E 2E7, Canada
}

Received 8 December 2009 / Accepted 7 April 2010

\section{ABSTRACT}

\begin{abstract}
Aims. We study the complex $\mathrm{H}_{2}$ outflows in the inner $300 \mathrm{AU}$ of the young triple star system $\mathrm{T}$ Tauri, with the goal of understanding the origin, excitation and evolution of the circumstellar matter.

Methods. Using high spatial resolution, integral-field spectroscopy in the $J$ and $K$ photometric bands from SINFONI/VLT, we trace the spatial distribution of $12 \mathrm{H}_{2}$ ro-vibrational emission lines, as well as one forbidden Fe II line. The ratio of line strengths provides a two-dimensional view of both the variable extinction and excitation temperature in this region, while the line-center velocities, coupled with previously published imagery, allow an assessment of the 3D space velocities and evolution of the outflows.

Results. Several spatially distinct flows - some with a bow shock structure - appear within 1".5 of the stars. Data taken two years apart clearly show the evolution of these flows. Some structures move and evolve, while others are stationary in the plane of the sky. The two-dimensional extinction map shows that the extinction between $\mathrm{T}$ Tau $\mathrm{N}$ and $\mathrm{T}$ Tau $\mathrm{S}$ is very high. In addition to being clumpy the extincting material forms part of a filament that extends to the east of the stars. In areas with strong line emission, the $v=1-0$ $S(1) / v=2-1 S(1)$ line ratio ranges from 8 to 20 , indicating that all of the observed $\mathrm{H}_{2}$ is shock excited. The outflows in the immediate vicinity of T Tau S span $\sim 270^{\circ}$ and are all blue-shifted, suggesting that they are produced by more than one star. We propose that $\mathrm{T}$ Tau N drives the east-west outflow, while T Tau Sa and T Tau Sb are the sources of the southeast-northwest and a previously undetected southwest outflow, respectively. There is a large spatial overlap between the [Fe II] line emission and previously measured UV fluorescent $\mathrm{H}_{2}$ emission, showing that both may be produced in $J$-shocks.
\end{abstract}

Key words. stars: individual: T Tauri - circumstellar matter - ISM: jets and outflows - stars: pre-main sequence

\section{Introduction}

$\mathrm{T}$ Tauri serves as the prototype of an entire class of pre-main sequence objects, yet is has over the last decades become increasingly obvious that the stellar system is very complex. T Tau ( $D=147.6$ pc, Loinard et al. 2007b) is a multiple system composed of an optically visible K0 star, T Tau N (Joy 1945; Beck et al. 2001), and a heavily extincted system, T Tau S (Dyck et al. 1982), approximately 0.7 south of the northern component. T Tau $\mathrm{S}$ is itself a binary with a separation of $\sim 50$ mas and $\mathrm{PA}=225^{\circ}$ at the time of discovery (Koresko 2000). The orbital period of the close binary is $\sim 21-28$ years and the components passed periastron in 1995 (Duchêne et al. 2006; Köhler 2008). At the time of observation the separation of the southern binary was 110 mas with $\mathrm{PA}=296^{\circ}$. While the western component of the binary, $\mathrm{T}$ Tau $\mathrm{Sb}$, appears to be a relatively normal $\mathrm{T}$ Tauri star residing behind an absorbing screen of $A_{V} \sim 15$ mag (Duchêne et al. 2005), the other object (T Tau Sa) remains an enigmatic source. T Tau Sa is the most massive object of the three $\left(2.3 M_{\odot}\right.$, Köhler 2008) and dominates the flux of the triple system at $\lambda>3 \mu \mathrm{m}$ (Herbst et al. 1997). Yet, it has never been detected at wavelengths short-wards of the $H$-band

* Based on observations collected at the European Southern Observatory, Paranal, Chile under the programme 60.A-9041(A).
(Herbst et al. 2007). T Tau Sa is highly variable in the nearinfrared (Beck et al. 2004). While it was brighter than T Tau Sb before $2000\left(K_{\mathrm{Sa}}=6.9, K_{\mathrm{Sb}}=8.8\right.$ in November 2000, Duchêne et al. 2002) it has undergone a rapid dimming and was the faintest of the three stars in December $2002\left(K_{\mathrm{Sa}}=9.8, K_{\mathrm{Sb}}=\right.$ 8.4, Duchêne et al. 2005). According to Duchêne et al. (2005) $\mathrm{T}$ Tau $\mathrm{Sa}$ is surrounded by an edge-on disk in addition to the absorbing screen in which $\mathrm{T}$ Tau $\mathrm{Sb}$ is embedded. The presence of an edge-on disk around $\mathrm{T}$ Tau $\mathrm{Sa}$ is supported by interferometric observations (Ratzka et al. 2009), while the orientation of the disk around T Tau Sb is largely unknown. T Tau N is on the other hand surrounded by a nearly face-on disk (Akeson et al. 1998; Gustafsson et al. 2008) which is therefore misaligned with the disk around T Tau Sa.

Studies of the environment of $\mathrm{T}$ Tau have also produced a number of surprises. Böhm \& Solf (1994) made the first subarcsecond study of the circumstellar environment and identified two bipolar outflows. They found a modest velocity outflow oriented southeast-northwest which they associated with T Tau S (still considered a single star at that time) and a second, high-velocity flow along the east-west direction associated with T Tau N. Bright arcs of forbidden line emission (Robberto et al. 1995) and near-infrared $\mathrm{H}_{2}$ line emission (Herbst et al. 1996, 1997) are found at scales of $\sim 2^{\prime \prime}-14^{\prime \prime}$, which reveals the complexity of the 
outflows in the T Tau system. At least 15 interlocking loops and filaments of $\mathrm{H}_{2}$ lie within $10^{\prime \prime}$ of the stars. Recent high spatial resolution images of $\mathrm{H}_{2}$ showed that the outflow pattern even in the immediate vicinity of the stars is highly complex (Herbst et al. 2007). Four bright arcs of $\mathrm{H}_{2}$ emission - associated with both the southeast-northwest flow and the east-west outflow are found within $1^{\prime \prime}$ of the stars. In contrast to Böhm \& Solf (1994), Herbst et al. (2007) associate the east-west flow with one of the components in the T Tau S binary. The southeastnorthwest outflow would then be produced by one of the two remaining stars.

In this paper we revisit the immediate vicinity of T Tau. We present new high spatial resolution data of the $\mathrm{H}_{2}$ line emission in the inner $2^{\prime \prime}$ which enables us to analyse the excitation mechanism of the $\mathrm{H}_{2}$ gas in unprecedented detail. With the new data together with the data of Herbst et al. (2007), we are able to follow the evolution of individual flows on a time base of two years. The derived proper motions present a unique possibility to pin-point the origin of the outflows in T Tau.

This paper is organised as follows. In Sect. 2 we describe the observations and data reduction and the main results are presented in Sect. 3. In Sect. 4 we discuss the implications on the ambient medium and the orientation of the stellar outflows. In Sect. 5 we give a summary of our findings.

\section{Observations}

T Tau was observed with the ESO-VLT as part of the SINFONI science verification program on the nights of 2004 October 30th and November 2nd. SINFONI is a near-infrared integral field spectrograph working in combination with adaptive optics (Eisenhauer et al. 2003). Observations of the region around the T Tau triple star system were obtained in the $K$-band using the 3 .'2 field of view optics (100 mas pixel scale) centered on the northern component and the 0.' 8 field of view optics ( 25 mas pixel scale) centered on the southern binary. In addition, we obtained $J$-band data using the 0. '8field of view centered on T Tau S, see Fig. 1. We used T Tau N $\left(m_{V}=9.6\right)$ as the adaptive optics guide star throughout, producing diffraction limited spatial resolution. The 2D image on the sky was sliced into 32 slitlets which were then dispersed onto a $2 \mathrm{k} \times 2 \mathrm{k}$ detector. The spectrograph provides a spectral resolution of 4000 in the $K$-band and 2000 in the $J$-band. Exposure times, number of co-adds and the total integration times are summarised in Table 1. The observations were carried out using a five-point nodding pattern and the resulting mosaics have slightly larger fields of view than the individual exposures (Fig. 1). Sky-frames with the same exposure times were obtained within the nodding cycle.

Data reduction and reconstruction of the 3D cubes were carried out using the SINFONI pipeline (version 1.3.0) provided by ESO. The 2D raw frames were corrected for sky background, flat field effects and optical distortions. Bad pixels and cosmic rays were identified and the frames were calibrated in wavelength. Then, the 3D cubes were constructed using calibration data of the locations of the slitlets on the detector. The cubes within a nodding cycle were aligned spatially and coadded spectral plane by spectral plane to create the final mosaic. Since the total exposure time is less in the outer regions of the mosaic than in the centre, we scaled the flux at all spatial points to the exposure time of a single frame. The final 3D cube stores the spatial information in the $x$ - and $y$-directions and the spectral information along the $z$-direction. To improve the signal-to-noise ratio, each spectral plane was smoothed with a 37.5 mas boxcar (the
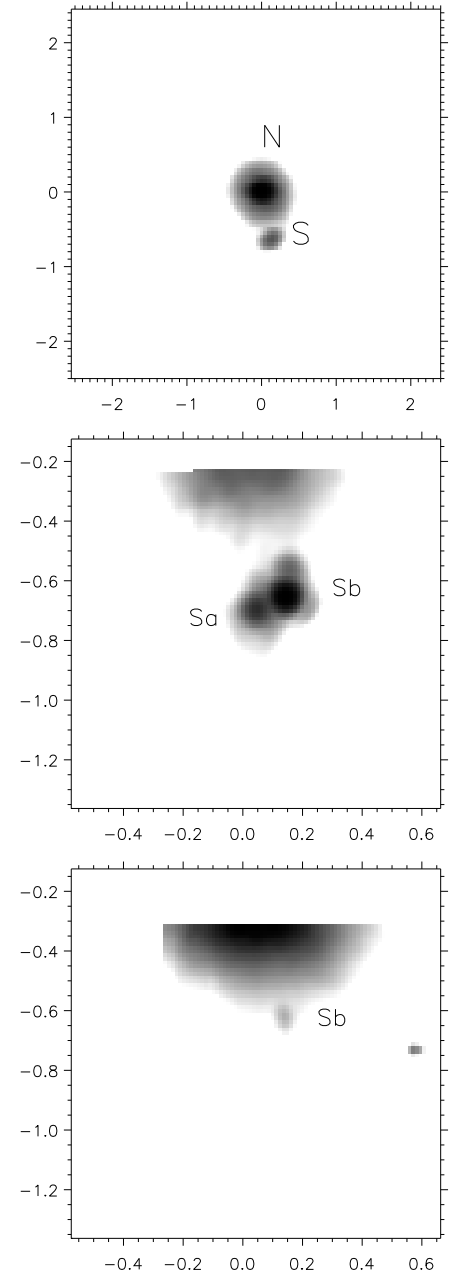

Fig. 1. Continuum images. Top: $K$-band broadband image computed by summing the spectral channels from 2.01-2.42 $\mu \mathrm{m}$ with the 100 mas pixel scale. Center: $K$-band broadband image with the 25 mas pixel scale. Bottom: $J$-band broadband image computed by summing the spectral channels at $1.13-1.37 \mu \mathrm{m}$ with the 25 mas pixel scale. Coordinates are offset from $\mathrm{T}$ Tau $\mathrm{N}\left(04^{\mathrm{h}} 21^{\mathrm{m}} 59^{\varsigma} .4,+19^{\circ} 32^{\prime} 06^{\prime \prime}\right)$ in arcseconds. The infrared companion, T Tau Sa, is not visible in the $J$-band. Scattered light from the northern component is seen in the center and bottom image.

Table 1. Observing log.

\begin{tabular}{cccccc}
\hline \hline No. & $\begin{array}{c}\text { Wavelength } \\
\text { Band }\end{array}$ & FoV $\left({ }^{\prime \prime}\right)$ & $\begin{array}{c}\text { Exposure } \\
\text { Time }(\mathrm{s})\end{array}$ & Co-adds & $\begin{array}{c}\text { Total Exp. } \\
\text { Time }(\mathrm{m})\end{array}$ \\
\hline 1 & $K$ & 3.2 & 3 & 20 & 30 \\
2 & $K$ & 0.8 & 120 & 1 & 30 \\
3 & $J$ & 0.8 & 900 & 1 & 60 \\
\hline
\end{tabular}

25 mas pixel scale data) or a 150 mas boxcar (the 100 mas pixel scale data) in the spatial domain. The resulting spatial resolution $(F W H M)$ is $180 \times 250$ mas in the 100 mas pixel scale data, $70 \times 70$ mas $(\mathrm{K})$ and $55 \times 65$ mas $(\mathrm{J})$ in the 25 mas data.

The B9 standard star Hip025657 was observed under the same conditions and similar airmass as $\mathrm{T}$ Tau and with the same instrumental setup in order to correct for atmospheric absorption. The spectrum was extracted after the data had been reduced following the same recipe as for the T Tau observations. The spectrum of Hip025657 is featureless in the $K$ and $J$-bands, except for the hydrogen $\mathrm{Br} \gamma$ and $\mathrm{Pa} \beta$ lines in absorption. We 
M. Gustafsson et al.: The origin, excitation, and evolution of subarcsecond outflows near T Tauri
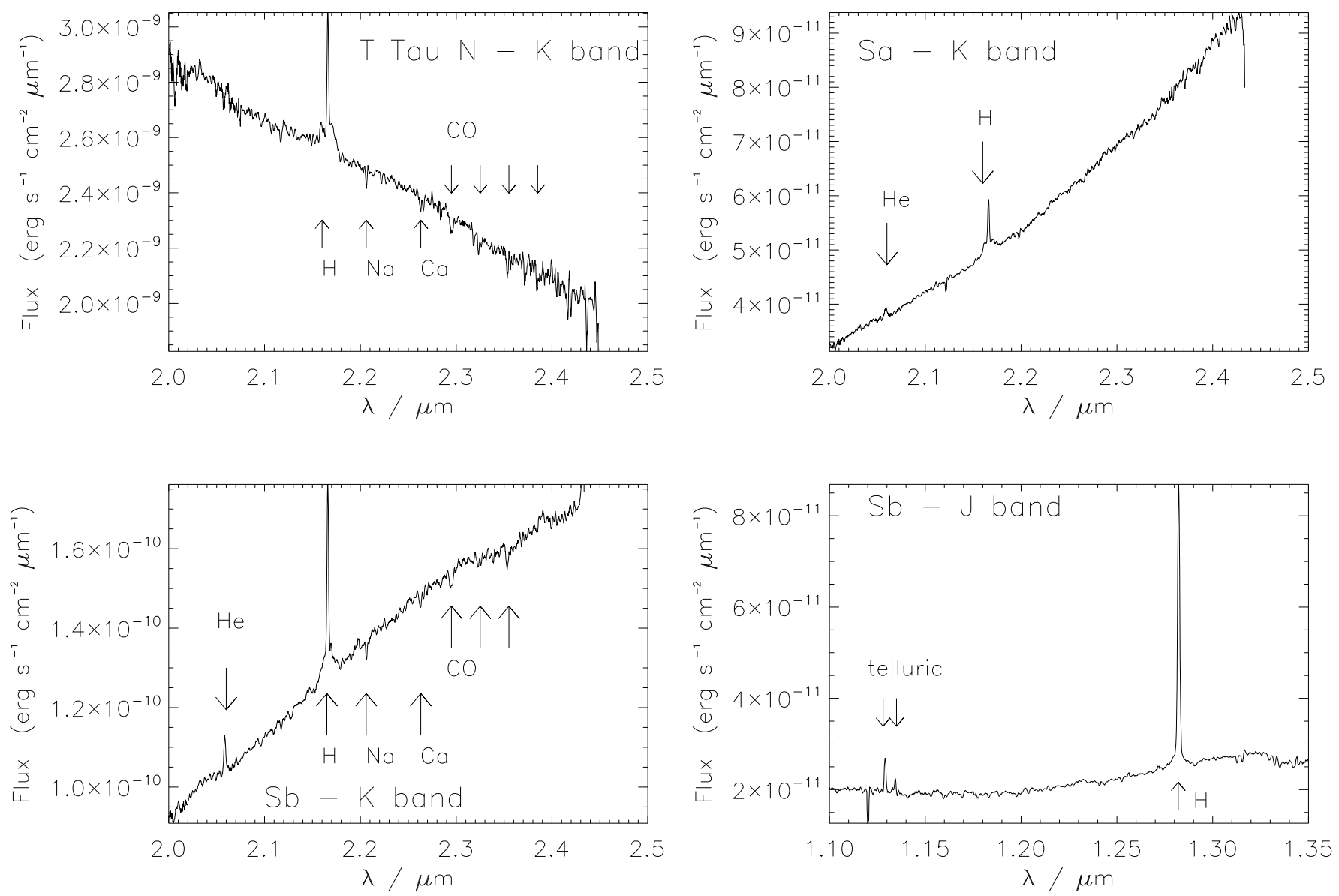

Fig. 2. Spectra of the three stars in the T Tau system.

removed those features and replaced them with a linear fit to the surrounding continuum. Subsequently, the spectrum was divided by a blackbody function of $T=11000 \mathrm{~K}$ and normalised. Dividing each spectrum of the science cube by the corrected standard star spectrum removed telluric absorption features in the T Tau spectra very effectively.

Flux calibration was also performed using Hip025657 $\left(m_{J}=\right.$ $\left.7.446, m_{K}=7.455\right)$. The conversion factor between counts s ${ }^{-1}$ and $\mathrm{erg} \mathrm{s}^{-1} \mathrm{~cm}^{-2} \mu \mathrm{m}^{-1} \mathrm{sr}^{-1}$ was found by dividing the published $K$-band flux of the calibrator star $\left(4.1 \times 10^{-7} \mathrm{erg} \mathrm{s}^{-1} \mathrm{~cm}^{-2} \mu \mathrm{m}^{-1}\right.$ $\times 10^{-m_{K} / 2.5}$ ) by the mean counts per second of its SINFONI spectrum within $1.94-2.45 \mu \mathrm{m}$ and dividing by the pixel area in steradians. Here we used the $K$-band zero-point flux from Campins et al. (1985). In the $J$-band we used the $J$-band zeropoint flux of $3.06 \times 10^{-6} \mathrm{erg} \mathrm{s}^{-1} \mathrm{~cm}^{-2} \mu \mathrm{m}^{-1}$ (Campins et al. 1985) to convert $\mathrm{m}_{J}$ into flux.

\section{Results}

\subsection{Simultaneous spectroscopy of the individual stars}

We have computed images of the $K$-band and the $J$-band continuum emission by summing the emission in all spectral channels between 2.01-2.42 $\mu \mathrm{m}$ and $1.13-1.37 \mu \mathrm{m}$, respectively (Fig. 1) In the large field of view $K$-band image, $\mathrm{T}$ Tau $\mathrm{N}$ and the southern binary are clearly seen, but the two binary components are not resolved. T Tau Sa and $\mathrm{Sb}$ are well separated in the 25 mas $K$-band image. Scattered light from $\mathrm{T}$ Tau $\mathrm{N}$ at the northern edge of the field of view is also evident in this image, as well as the first Airy ring of the PSF of $\mathrm{T}$ Tau Sa and Sb. The Airy ring is somewhat deformed due to quasi-static aberrations in the optical system with two peaks north and southwest of the main component. The PSF of the reference star shows the same wings north and southwest of the star. We used the PSF of the reference star to subtract the signal from $\mathrm{T}$ Tau $\mathrm{Sb}$, which resulted in a very clean image of the PSF from T Tau Sa. This procedure allowed us to isolate and extract the spectra of the two components with minimal blending of the signals. In the $J$-band image, only $\mathrm{T}$ Tau $\mathrm{Sb}$ is detected while Sa remains undetected at wavelengths shorter than $H$-band (Herbst et al. 2007).

Broad-band photometry of the stellar components was performed using the integrated continuum image (Fig. 1). The $K$-band magnitude of $\mathrm{T}$ Tau $\mathrm{N}, \mathrm{Sa}$ and $\mathrm{Sb}$ are estimated to $5.4 \pm 0.1 \mathrm{mag}, 9.6 \pm 0.2 \mathrm{mag}, 8.7 \pm 0.1 \mathrm{mag}$, respectively. In the $J$-band the magnitude of T Tau Sb is $12.2 \pm 0.7 \mathrm{mag}$. The large uncertainty on the $J$-band flux of T Tau $\mathrm{Sb}$ is due to the poor flux ratio between the scattered light from $\mathrm{T}$ Tau $\mathrm{N}$ and the faint source. At this epoch, the Sb component is much brighter in the $K$-band than the Sa component, showing that Sa continues to be the faintest component after the rapid dimming that it has undergone between 2000 and 2002 (Duchêne et al. 2005).

Spectra of the three stellar components extracted from the data cubes appear in Fig. 2. The spectrum of $\mathrm{T}$ Tau $\mathrm{N}$ is dominated by the $\mathrm{Br} \gamma$ emission line at $2.166 \mu \mathrm{m}$. The line appears asymmetric with very broad wings, but that is mainly due to the lack of correction for the telluric absorption in this spectral region. The telluric standard star has a strong Br $\gamma$ absorption feature and therefore it was not possible to remove telluric features within a spectral range of 2.16-2.17 $\mu \mathrm{m}$ (see Sect. 2). 

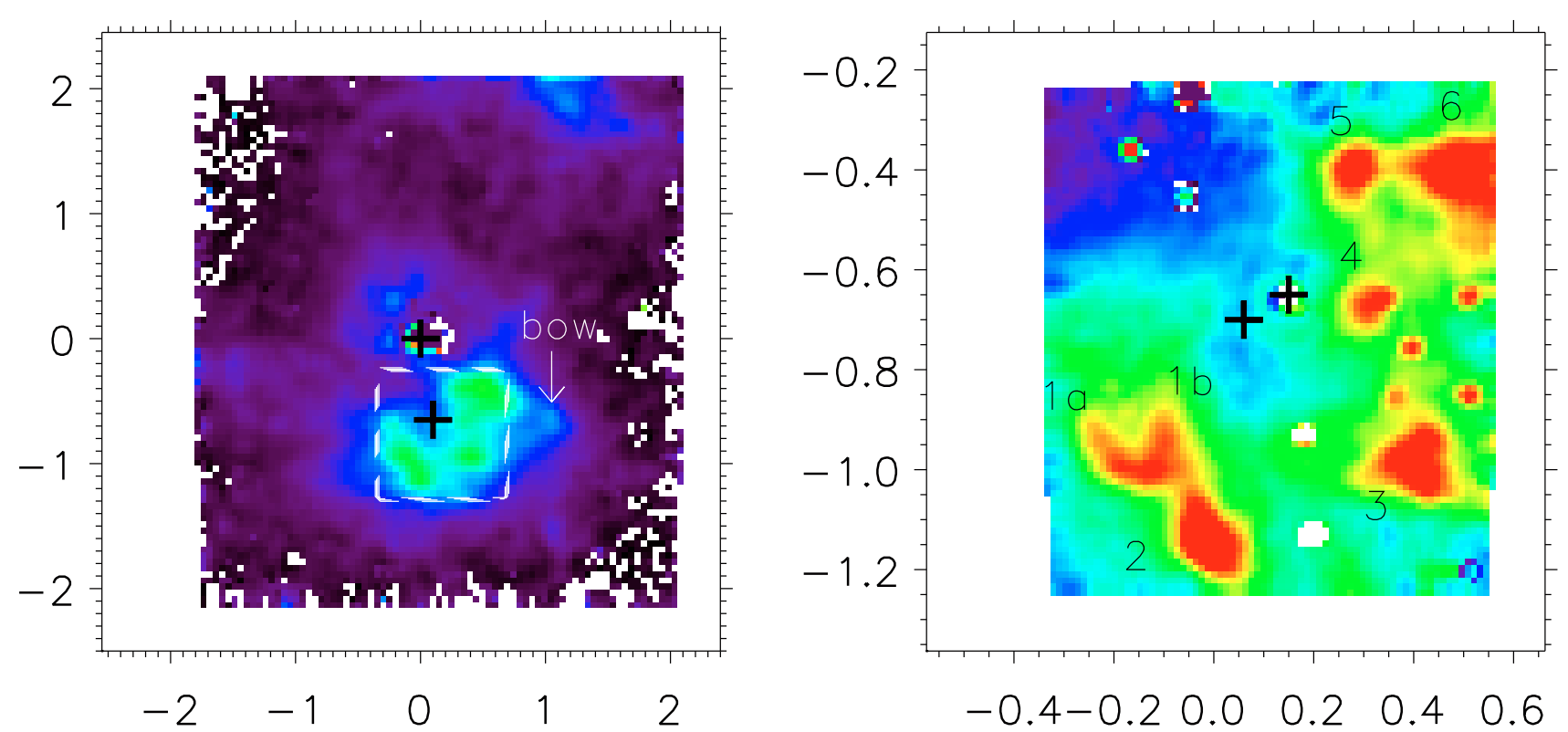

Fig. 3. Images of $\mathrm{H}_{2} v=1-0 S(1)$ emission. Left -100 mas pixel scale, Right -25 mas pixel scale. The colourbars indicate the flux level in $\mathrm{erg} \mathrm{s}^{-1} \mathrm{~cm}^{-2} \mathrm{sr}^{-1}$. The spatial coverage of the small field of view (right) is indicated by the white box in the large field of view image to the left. The position of the stars are marked with black crosses.

The spectrum also contains several photospheric features, including $\mathrm{NaI}, \mathrm{CaI}$ and the $\mathrm{CO} \Delta v=2$ bandheads as previously reported by Beck et al. (2004).

The infrared companion, $\mathrm{T}$ Tau Sa, has a very red continuum slope. The spectrum is completely featureless except for $\mathrm{Br} \gamma$ emission and the recombination line of helium at $2.058 \mu \mathrm{m}$ first identified in T Tau Sa/Sb by Herbst et al. (2007). Neutral helium is ionised by EUV radiation. We studied the spatial extent of the He I emission by analysing the line to continuum ratio. We did not find any evidence of the He I emission being spatial extended. The presence of HeI emission and the fact that the emission is not extended, thus indicates that, while the EUV radiation from the star is strong enough to ionise helium significantly, the helium ionisation front is situated close to the stellar surface. The continuum slope of T Tau Sb is less red than that of T Tau Sa and both the $\mathrm{Br} \gamma$ and the He I emission lines are stronger than in the infrared companion. The He I emission line flux has been measured to $0.51 \times 10^{-17} \mathrm{~W} \mathrm{~m}^{-2}$ and $2.0 \times 10^{-17} \mathrm{~W} \mathrm{~m}^{-2}$ in T Tau Sa and $\mathrm{Sb}$, respectively. Photospheric features from $\mathrm{Na}, \mathrm{Ca}$ and $\mathrm{CO}$ are also seen in the spectrum of T Tau Sb. The $J$-band spectrum of $\mathrm{T} \mathrm{Tau} \mathrm{Sb}$ is featureless except for $\mathrm{Pa} \beta$ in emission.

\section{2. $\mathrm{H}_{2}$ lines}

Within the $K$-band data cubes, several $\mathrm{H}_{2}$ rovibrational emission lines are clearly detected. $\mathrm{No}_{2}$ emission was detected in the $J$-band data where the $\mathrm{H}_{2}$ lines are intrinsically weaker than those in the $K$-band. Thus, the following only relates to $K$-band lines.

Emission from the $\mathrm{H}_{2} v=1-0 \mathrm{~S}(1)$ line at $2.1218 \mu \mathrm{m}$ is detected everywhere within $2^{\prime \prime}$ from T Tau N (Herbst et al. 1996), see Fig. 3. The strongest emission is concentrated in the immediate surroundings of T Tau $\mathrm{S}$ within a radius of $0 .{ }^{\prime} 8$ and strong emission is found as close as 0.1 ( $~ 14$ AU) from T Tau Sb. A number of the strongly emitting regions have a morphology resembling bow-shocks. To the north in the large field of view the southern tip of the emission knot called T Tau NW (Herbst et al. 1996 ) is seen. No molecular hydrogen emission was found in any of the three stellar components, confirming that the line emission seen in lower spatial resolution spectra of the stars stems from excited gas in the vicinity of the stars (Beck et al. 2004; Duchêne et al. 2005). In Fig. 3 we have marked seven distinct emission regions, some of which clearly resemble bow shocks.

With the SINFONI datacube, we can construct line maps of all detected $\mathrm{H}_{2}$ lines. We calculate the line brightness based on fits of gaussian profiles $\left(F=A \exp \left[-\left(\lambda-\lambda_{0}\right)^{2} / 2 \sigma^{2}\right]+b+c \lambda\right)$ to the line profiles. This procedure assumes that only one spectral peak is present and includes a linear fit of the continuum. Given the high spatial resolution and the spectral resolution of $\sim 75 \mathrm{~km} \mathrm{~s}^{-1}$ of SINFONI, this is always the case in our data. The flux is given by the integral of the emission profile $\sqrt{2 \pi} A \sigma$ and the formal uncertainty is $\Delta F=\left[2 \pi\left(\sigma^{2} \Delta A^{2}+A^{2} \Delta \sigma^{2}\right)\right]^{1 / 2}$, where $\Delta A$ and $\Delta \sigma$ are those changes in the $A$ and $\sigma$ parameters which increase the $\chi^{2}$ per degree of freedom by 1 (Bevington 1969). The uncertainty on the line flux depends on line strength. In strongly emitting regions the relative uncertainty is typically around $10 \%$, whereas the uncertainty reaches $\sim 50 \%$ in weakly emitting regions.

Detection of weaker excitation lines is generally limited to regions of strong $v=1-0 S$ (1) emission, although the flux ratios of $\mathrm{H}_{2}$ lines are found to vary with position. That is, in regions where the $v=1-0 S(1)$ emission is equally strong, the emission in other lines may vary. The $K$-band spectrum of one of the regions with the strongest $v=1-0 S$ (1) emission (labelled 2 in Fig. 3) shows clear detections of almost all $\mathrm{H}_{2}$ rovibrational lines from the $v=1-0$ and $v=2-1$ bands within the $K$-band (see 
M. Gustafsson et al.: The origin, excitation, and evolution of subarcsecond outflows near T Tauri

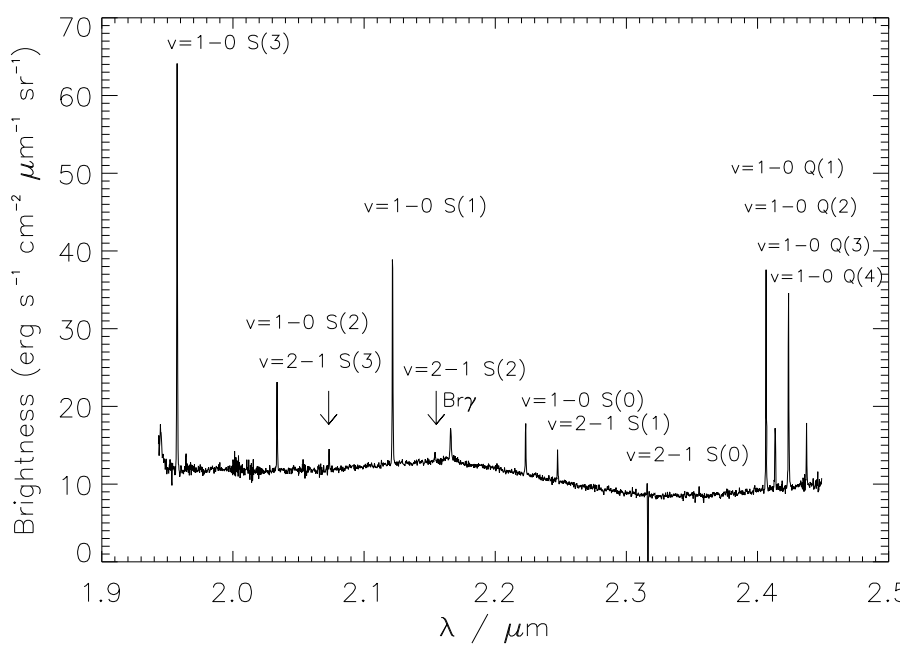

Fig. 4. $K$-band spectrum of the strongly $\mathrm{H}_{2}$ emitting region southeast of $\mathrm{T}$ Tau $\mathrm{S}$ at $\left(0^{\prime \prime},-1{ }^{\prime \prime} 15\right)$ called 2 in Fig. 3 . A wealth of $\mathrm{H}_{2}$ lines from the $v=1-0$ and $2-1$ bands is detected. The $\mathrm{Br} \gamma$ line is due to scattered light from the nearby stars.

Fig. 4). An exception is the $v=2-1 \mathrm{~S}(4)$ line at $2.00 \mu \mathrm{m}$ which, in addition to being weak, is located in a spectral region where correction for atmospheric $\mathrm{H}_{2} \mathrm{O}$ and $\mathrm{OH}$ lines is known to be problematic. Note also that the $v=1-0 \mathrm{~S}(3)$ line and the $v=1-0$ $Q$-branch are located in spectral regions with strong atmospheric absorption and care should be exercised when using the derived flux of these lines. Emission from the hydrogen $\mathrm{Br} \gamma$ line is also seen in Fig. 4. In order to locate the origin of the Br $\gamma$ emission we inspected the spatial distribution of the emission. It turned out that the line to continuum ratio remains constant throughout the region. We therefore conclude that the $\operatorname{Br} \gamma$ emission is not produced locally, but is caused by scattered light from the stars which permeates the entire region.

The strength of the line emission of all the detected $\mathrm{H}_{2}$ lines from the seven regions marked in Fig. 3 is listed in Table 2. The recorded line emission is the average value in the region in which the line brightness is larger than half of the local maximum brightness. Table 2 also lists the sizes of the emitting regions.

\subsubsection{Extinction}

The extinction along the line-of-sight can be estimated by considering the flux of two transitions arising from the same upper level. For an optically thin transition at wavelength $\lambda$, the line intensity $I$ is given by

$I=\frac{A_{E} h c}{4 \pi \lambda} N_{\mathrm{u}} 10^{-A_{\lambda} / 2.5}$

where $A_{\mathrm{E}}$ is the Einstein A coefficient, $N_{\mathrm{u}}$ is the number of molecules in the upper state, and $A_{\lambda}$ is the extinction. The ratio of two lines with the same upper level is

$\frac{I_{1}}{I_{2}}=\frac{A_{E 1} \lambda_{2}}{A_{E 2} \lambda_{1}} 10^{-\left(A_{\lambda_{1}}-A_{\lambda_{2}}\right) / 2.5}$,

Standard interstellar extinction laws can be used to convert $A_{\lambda_{1}}-$ $A_{\lambda_{2}}$ into $A_{V}$. We use $A_{\lambda} / A_{K}=(\lambda / 2.2 \mu \mathrm{m})^{-1.7}$ and $A_{K} / A_{V}=0.108$ (Mathis 1990).

In our data, two sets of lines share the same upper level. The $v=1-0 S(1)$ and the $v=1-0 \mathrm{Q}(3)$ lines both arise from the $v=1, J=3$ level and the $v=1-0 \mathrm{~S}(0)$ and the $v=1-0 \mathrm{Q}(2)$ lines both arise from the $v=1, J=2$ level. The $Q$-branch is located in a spectral region where atmospheric correction is known to be difficult, but by careful inspection of the corrected and the uncorrected spectrum in the $2.40-2.44 \mu \mathrm{m}$ interval, we find that the telluric absorption lines located in the continuum between the $\mathrm{H}_{2}$ lines are very well removed. That is, the continuum is featureless as it should be with no or a few, weak traces of telluric lines after the correction. We assume that the correction for atmospheric absorption at the position of $\mathrm{H}_{2}$ lines is equally good and conclude that in these data the derived line flux is not heavily biased by absorption. However, the atmospheric correction does introduce an uncertainty to the derived line flux which we estimate to be $\sim 10 \%$, but as this is generally smaller than the formal uncertainty of the line flux we choose to ignore it.

The visual extinction $A_{V}$ is estimated as

$A_{V, 31}=108 \log \left(1.426 \frac{I_{Q(3)}}{I_{S(1)}}\right)$ and
$A_{V, 20}=182 \log \left(0.906 \frac{I_{Q(2)}}{I_{S(0)}}\right)$,

where we have used the Einstein A coefficients from Turner et al. (1977).

In Fig. 5 we plot $A_{V, 31}$ vs. $A_{V, 20}$ for all spatial points in the 25 mas data. The two estimates of the extinction do not agree very well. The majority of points lie far from the $A_{V, 31}=A_{V, 20}$ line. While values of $A_{V, 31}$ are found in the interval between 0 and 30 , the $A_{V, 20}$ values show a much larger spread ranging from -60 to 60 . The large spread and the unphysical negative values reflects the fact that the $v=1-0 \mathrm{~S}(0)$ and the $v=1-0 \mathrm{Q}(2)$ lines are much weaker than the $v=1-0 S(1)$ and the $v=1-0$ $\mathrm{Q}(3)$ lines and are therefore associated with much larger relative uncertainty. For both $A_{V, 31}$ and $A_{V, 20}$ we calculated the $A_{V}$ interval that is spanned when the flux of the lines is allowed to vary $\pm 1 \sigma$. This analysis showed three things: i) within the uncertainty, the $A_{V, 20}$ value is always consistent with being positive; ii) for all pixels the $A_{V, 31}$ interval is smaller than the $A_{V, 20}$ interval and the $A_{V, 31}$ interval is always contained within the $A_{V, 20}$ interval; iii) even the $A_{V, 31}$ value, which is derived from two of the strongest $\mathrm{H}_{2}$ lines, spans a large range of values. The interval varies from $A_{V, 31}=[0,15]$ in strongly emitting regions, where the relative uncertainty on the line flux is typically $10 \%$, to $[-30,80]$ in weak regions where the relative uncertainty is as high as $\sim 50 \%$. For $A_{V, 20}$ the interval is typically $A_{V, 20}=[-15,65]$ in strongly emitting regions.

This analysis shows that the derivation of extinction from line ratios is highly dependent on high signal-to-noise and high accuracy of flux determination. Our results clearly demonstrate the difficulty in obtaining reliable estimates of the extinction. Even a modest flux uncertainty of $10 \%$ results in a large uncertainty of the extinction value.

With this in mind, we use the $A_{V}$ values derived from the strongest lines, that is $A_{V, 31}$, as the best estimate of the extinction towards T Tau. Maps of $A_{V, 31}$ are shown in Fig. 6 and comparison with the contours of $\mathrm{H}_{2}$ brightness gives an impression of the uncertainty, keeping the above mentioned uncertainty limits in mind. Due to the large uncertainty in the values of $A_{V}$ we have not corrected the $\mathrm{H}_{2}$ line flux for extinction in any line.

Even though the absolute values of the extinction are highly uncertain, there is reason to believe that the variations in $\mathrm{A}_{V}$ are real. Variations of $15 \mathrm{mag} A_{V}$ are found in regions with the same $v=1-0 S(1)$ brightness level. This means that $A_{V}$ is not correlated with $\mathrm{H}_{2}$ line brightness. Furthermore, the overlapping regions in the 100 mas and 25 mas map in Fig. 6 show the 
Table 2. Line emission (in $10^{-2} \mathrm{erg} \mathrm{s}^{-1} \mathrm{~cm}^{-2} \mathrm{sr}^{-1}$ ), radial velocity with respect to the rest velocity of $\mathrm{T}$ Tau Sa, tangential velocity, inclination angle, excitation temperature and size of the seven regions marked in Fig. 3.

\begin{tabular}{ccccccccc}
\hline \hline Line & $\lambda(\mu \mathrm{m})$ & $1 \mathrm{a}$ & $1 \mathrm{~b}$ & 2 & 3 & 4 & 5 & 6 \\
\hline$v=1-0 \mathrm{~S}(3)$ & 1.9576 & 1.7 & 1.5 & 2.5 & 1.6 & 1.4 & 1.7 & 1.2 \\
$v=1-0 \mathrm{~S}(2)$ & 2.0338 & 0.40 & 0.39 & 0.50 & 0.41 & $<1.2^{a}$ & 0.45 & 0.40 \\
$v=2-1 \mathrm{~S}(3)$ & 2.0729 & $<0.16^{a}$ & $<0.097^{a}$ & 0.10 & 0.083 & $<0.14^{a}$ & $<0.075^{a}$ & $<0.17^{a}$ \\
$v=1-0 \mathrm{~S}(1)$ & 2.1218 & 1.1 & 1.1 & 1.4 & 1.2 & 1.3 & 1.3 & 1.1 \\
$v=2-1 \mathrm{~S}(2)$ & 2.1542 & 0.058 & 0.056 & $<0.094^{a}$ & $<0.047^{a}$ & $<0.10^{a}$ & $<0.072^{a}$ & $<0.078^{a}$ \\
$v=1-0 \mathrm{~S}(0)$ & 2.2235 & 0.28 & 0.27 & 0.31 & 0.25 & 0.32 & 0.24 & 0.31 \\
$v=2-1 \mathrm{~S}(1)$ & 2.2477 & 0.11 & 0.13 & 0.14 & 0.11 & $<0.11^{a}$ & $<0.077^{a}$ & $<0.090^{a}$ \\
$v=2-1 \mathrm{~S}(0)$ & 2.3556 & $<0.045^{a}$ & $<0.044^{a}$ & $<0.058^{a}$ & $<0.054^{a}$ & $<0.10^{a}$ & $<0.083^{a}$ & $<0.15^{a}$ \\
$v=1-0 \mathrm{Q}(1)$ & 2.4066 & 1.1 & 1.1 & 1.2 & 1.3 & 1.5 & 1.3 & 1.4 \\
$v=1-0 \mathrm{Q}(2)$ & 2.4134 & 0.32 & 0.33 & 0.40 & 0.36 & 0.40 & 0.39 & 0.39 \\
$v=1-0 \mathrm{Q}(3)$ & 2.4237 & 0.96 & 0.98 & 1.1 & 1.1 & 1.2 & 1.2 & 1.1 \\
$v=1-0 \mathrm{Q}(4)$ & 2.4375 & 0.55 & 0.30 & 0.32 & 0.24 & 0.20 & 0.20 & $<0.21^{a}$ \\
rad. vel. (km s $\left.{ }^{-1}\right)$ & & -22 & -19 & -21 & -17 & -9 & -10 & -8 \\
tang. vel. (km s $\left.{ }^{-1}\right)$ & & 53 & & & 32 & - & - & \\
inclination ( $\left.{ }^{\circ}\right)$ & & 70 & & & 60 & & & 1100 \\
Ex. Temp. (K) & & 2000 & 2000 & 2100 & 1900 & 1100 & 1300 \\
size (AU) & & 21 & 21 & 26 & 37 & 25 & 25 & 25 \\
\hline
\end{tabular}

Notes. ${ }^{a} 2 \sigma$ upper limit.

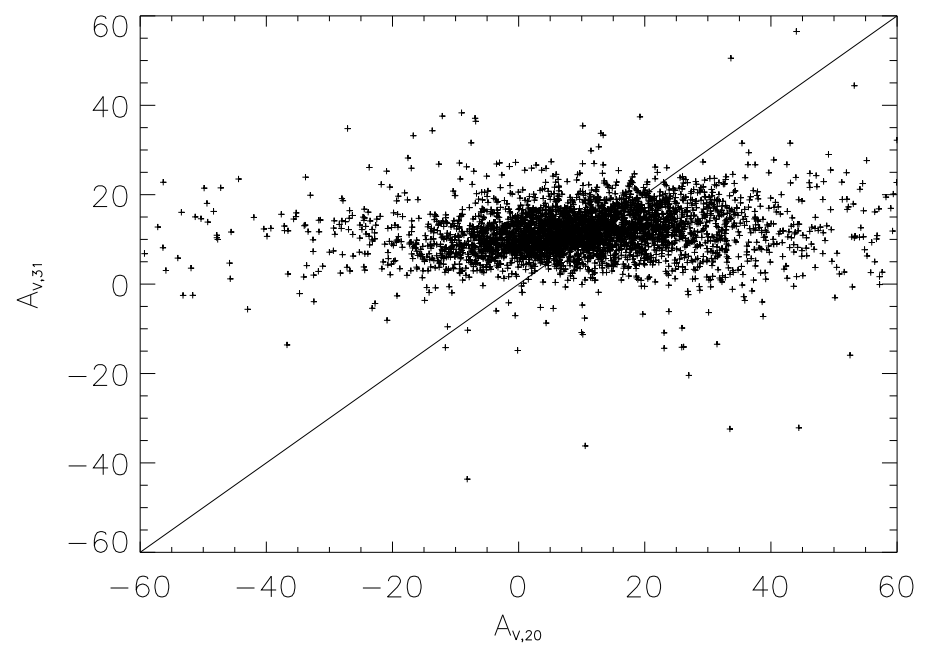

Fig. 5. $A_{V, 31}$ vs $A_{V, 20}$ calculated for each spatial point in the 25 mas data. The solid line represents $A_{V, 31}=A_{V, 20}$.

same $A_{V}$ structure around $\mathrm{T}$ Tau $\mathrm{S}$. In addition, our 100 mas pixel scale image (Fig. 6 left) agrees very well with the recently published extinction map of Beck et al. (2008). In the 100 mas image (Fig. 6 left) the obscuring material between the north and south components is seen to be part of a filament that continues to the east of T Tau. South of T Tau S, the extinction is lower by $A_{V} \sim 15$. If the $A_{V}$ variations are real it implies that the circumstellar material is very clumpy. For a discussion of the implications see Sect. 4.

\subsubsection{The $v=1-0 S(1) / v=2-1 S(1)$ line ratio}

Near-infrared rovibrational emission lines from $\mathrm{H}_{2}$ arise in a number of physical processes, the most common of which are shock excitation and ultraviolet fluorescence. Line ratios of different $\mathrm{H}_{2}$ lines can be used to discriminate between these two mechanisms. The excitation mechanism at play is most clearly distinguished by comparing the emission in the $v=1-0$ to the emission in the $v=2-1$ vibrational band. Fluorescence results in stronger excitation to the high energy band than shock excitation. Here, we use the $v=2-1 S$ (1) line as a representative for the emission in the $v=2-1$ band.

Figure 7 shows the distribution of the $v=2-1 S(1)$ line together with contours of the $v=1-0 S(1)$ line. The $v=2-1$ $S(1)$ emission is only detected around T Tau $S$ and in the NW knot (Fig. 7 left). The spatial distribution of the $v=2-1 S(1)$ emission is not identical to that of the $v=1-0 S(1)$ emission. This becomes clearer in the right-hand side of the figure which shows the distribution of the $v=2-1 S(1)$ line in the 25 mas pixel scale data. Emission from the $v=2-1 S(1)$ transition is only detected southwest and southeast of T Tau S. Surprisingly, no $v=2-1 S(1)$ emission is found northwest of the southern binary, even though the $v=1-0 S(1)$ emission is strong in that region. Figure 8 shows the corresponding $v=1-0 S(1) / v=2-1$ $S$ (1) line ratio map.

The different distribution of $v=1-0 S(1)$ and $v=2-1 S(1)$ emission results in a high variability in the line ratio. In Fig. 8 right, the lowest value of $\sim 8$ is found southwest and southeast of T Tau $\mathrm{S}$ and is coincident with peaks in the $v=1-0 S(1)$ emission. Closer to $\mathrm{T}$ Tau $\mathrm{S}$ and northwest of the binary, very little $v=2-1 S(1)$ emission is detected and the line ratio reaches values of $\sim 15-20$. A particular noteworthy feature is the flow at $\left(-0 .{ }^{\prime} 1,-1^{\prime \prime} 0\right)$ (labelled $1 \mathrm{a} / 1 \mathrm{~b}$ in Fig. 3) which in the $v=1-0$ $S$ (1) line appears to be one coherent entity with the shape of a horseshoe. In the $v=2-1 S$ (1) line, the western side of the horseshoe (1b) closest to T Tau $\mathrm{S}$ is weaker than the eastern side (1a) and in the line ratio map there is no evidence of a horseshoe shape. The horseshoe could therefore be the result of two flows instead of a single coherent flow.

The strength of the line emission from all the detected $\mathrm{H}_{2}$ lines in the $K$-band extracted from the seven distinct emission features marked in Fig. 3 is listed in Table 2. In all seven regions the $v=1-0 S(1) / v=2-1 S(1)$ line ratio is larger than 10 . These values are only compatible with shock excitation, since fluorescence produces values of $\sim 2$ (Black \& van Dishoeck 1987). In the following sections, we work with the hypothesis that all - or at least most - of the observed emission is caused by shocks. 
M. Gustafsson et al.: The origin, excitation, and evolution of subarcsecond outflows near T Tauri
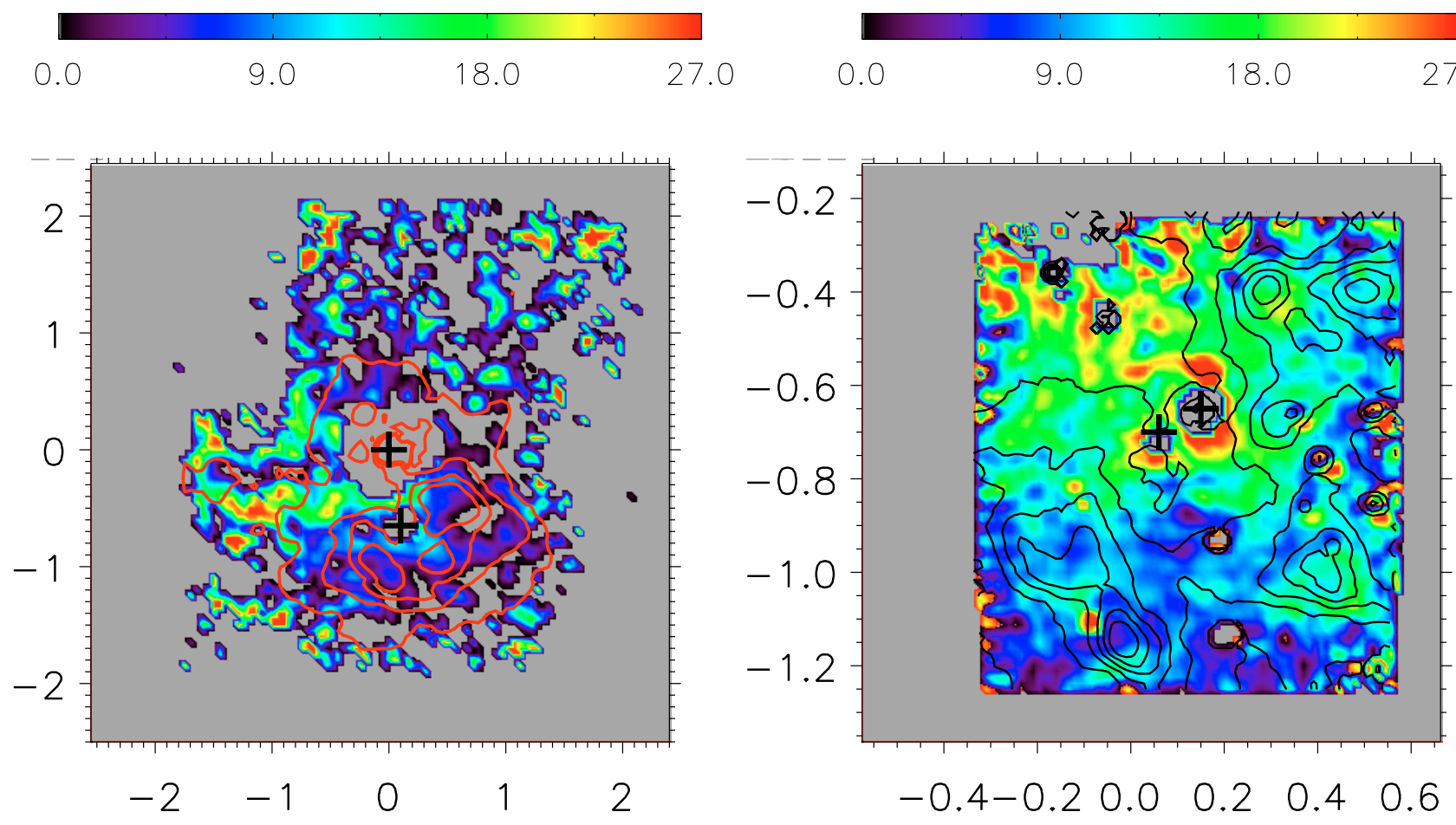

Fig. 6. Maps of extinction. The estimate $A_{V, 31}$ is shown for the 100 mas (left) and 25 mas (right) pixel scale. The colourbars indicate the magnitude of $A_{V}$. Only regions in which the $\mathrm{H}_{2} v=1-0 S(1)$ line is detected at a $8 \sigma$ level (left) or a $3 \sigma$ level (right) are displayed. Contours represent $v=1-0$ $S$ (1) brightness.
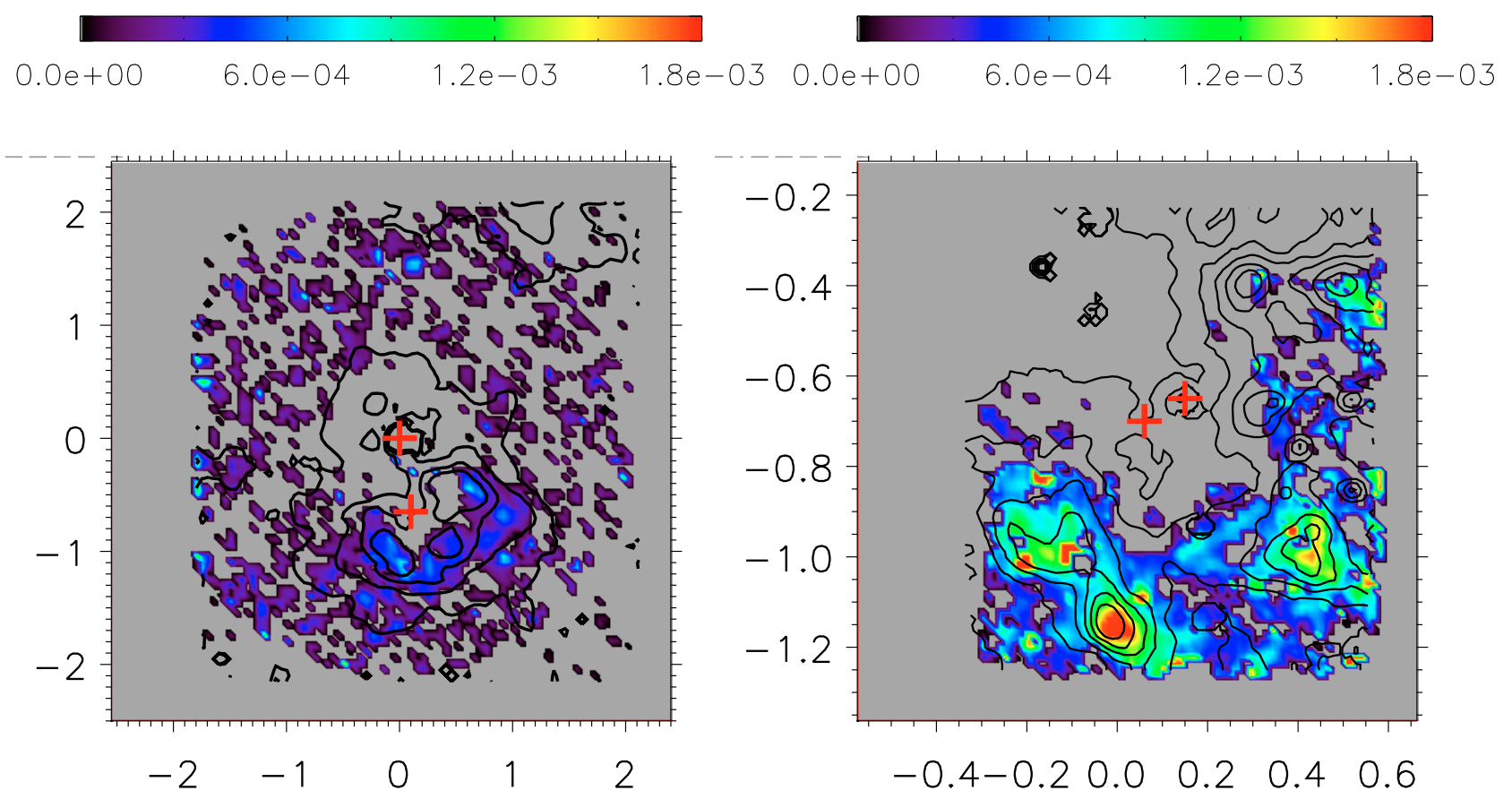

Fig. 7. $\mathrm{H}_{2} v=2-1 S(1)$ brightness in $\mathrm{erg} \mathrm{s}^{-1} \mathrm{~cm}^{-2} \mathrm{sr}^{-1}$. Left: 100 mas pixel scale, right: 25 mas pixel scale. Contours outline the $v=1-0 S(1)$ brightness. Only regions in which the $v=2-1 S(1)$ line is detected at a $2 \sigma$ level are displayed.

\subsubsection{Excitation temperature}

In the case of shock excitation, a super-Alfvenic shock wave rapidly heats the gas to temperatures of $>1000 \mathrm{~K}$. The temperature reached depends on the type of shock ( $J$-shock or $C$-shock) as well as the shock velocity and the pre-shock conditions in the medium (Kristensen et al. 2007).
Using the line strengths of all available $\mathrm{H}_{2}$ lines, we calculate the excitation temperature in every spatial pixel. The excitation temperature is the temperature that reproduces the observed line ratios, assuming local thermodynamic equilibrium, LTE. In LTE conditions, the column density of level $(v, J)$ is

$N_{v, J}=\frac{N_{\text {tot }}}{Z(T)} g_{v, J} \exp \left(-E_{v, J} / T\right)$ 

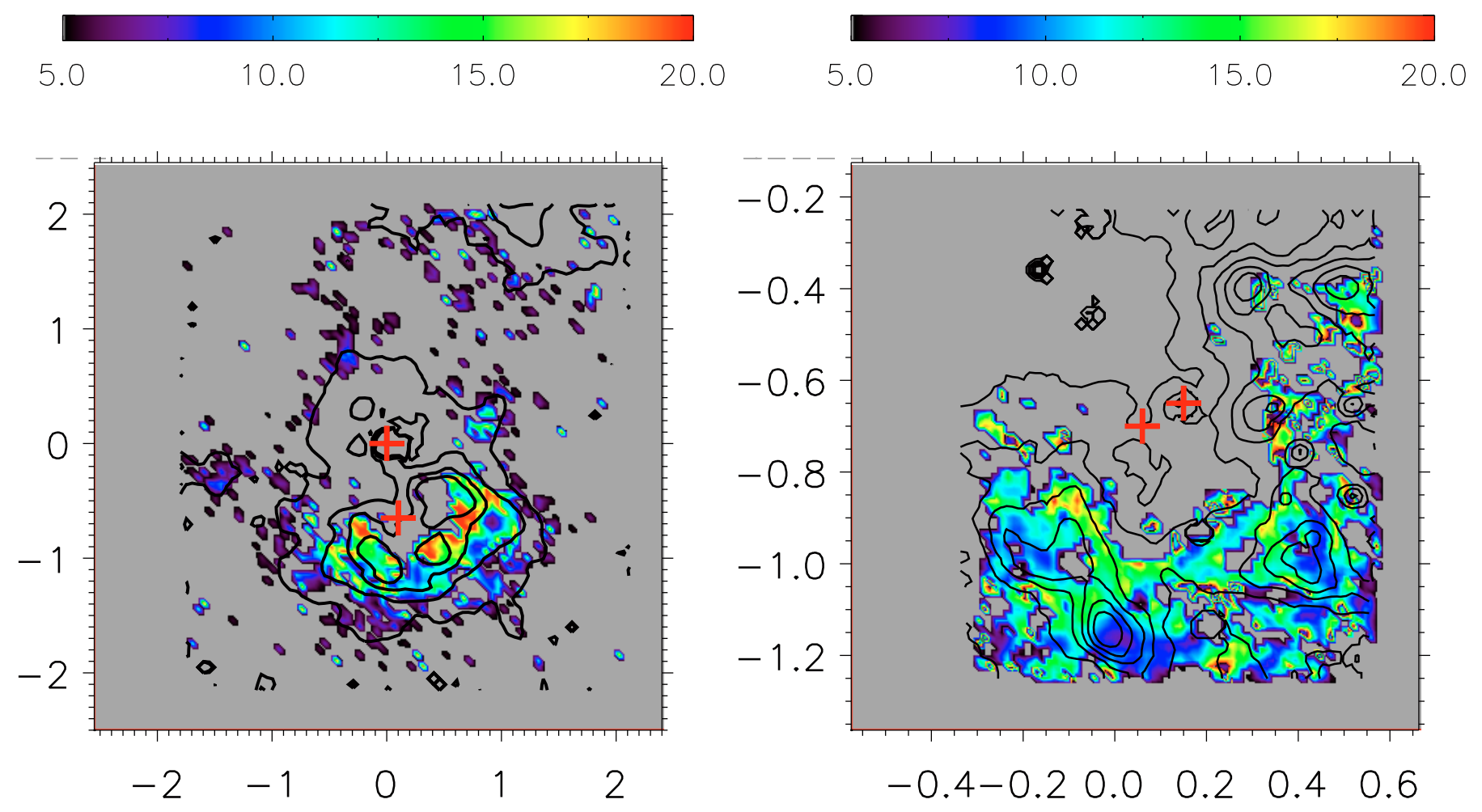

Fig. 8. The ratio of $\mathrm{H}_{2} v=1-0 S(1)$ to $v=2-1 S(1)$ line emission. Left: 100 mas pixel scale, right: 25 mas pixel scale. Contours outline the $v=1-0 S(1)$ brightness. Only regions in which the $v=2-1 S(1)$ line is detected at a $2 \sigma$ level are displayed.

where $N_{\text {tot }}$ is the total column density, $Z(T)$ is the partition function, $g_{v, J}$ is the degeneracy of the level, $E_{v, J}$ is the level energy expressed in $K$, and $T$ is the excitation temperature. Using the $(v, J)=(1,3)$ level as reference we obtain

$T=-\frac{E_{v, J}-E_{1,3}}{\ln \left(\frac{N_{v, J} g_{1,3}}{N_{1,3} g_{v, J}}\right)}$.

In Fig. 9, we plot $\ln \left(N_{v, J} g_{1,3} /\left(N_{1,3} g_{v, J}\right)\right)$ against the energy difference for a spatial position associated with bright $v=1-0 S(1)$ emission SSE of T Tau S in the flow labelled 2 (Figs. 3 and 4). The degeneracy, $g_{v, J}$, is $(2 J+1)$ for even $J$ states (para-hydrogen) and $3(2 J+1)$ for odd $J$ states (ortho-hydrogen). Here, we assume that the ortho/para ratio is 3 . Deviations from the equilibrium value of 3 would result in a displacement of the ortho and para points in the excitation diagram and there is no evidence for such displacements.

The line ratios in Fig. 9 are well represented within the uncertainties by a single linear fit with an excitation temperature of $1990 \mathrm{~K}$. The only exception is the $v=1-0 \mathrm{~S}(3)$ line which lies significantly displaced from the linear fit. We ascribe this to poor correction for telluric absorption in the $1.95 \mu \mathrm{m}$ spectral region and ignore the $v=1-0 \mathrm{~S}(3)$ line in all further analysis. Note that the derived column densities of the $Q$-branch lines (Fig. 9) agree within the uncertainties with the column densities of the corresponding $S$-branch lines which share the same upper level. This is very comforting and supports our earlier conclusion that the $Q$-branch is not significantly affected by errors in the correction for atmospheric absorption. Note also that no correction for extinction has been applied to the line strengths. However, since all lines lie in the $K$-band, any differential effect of extinction would be small, $\Delta A_{\lambda}=25 \%$ from $2.0 \mu \mathrm{m}$ to $2.4 \mu \mathrm{m}$. This is demonstrated in Fig. 9 where the column densities of both uncorrected and dereddened data are plotted. The dereddening corresponds

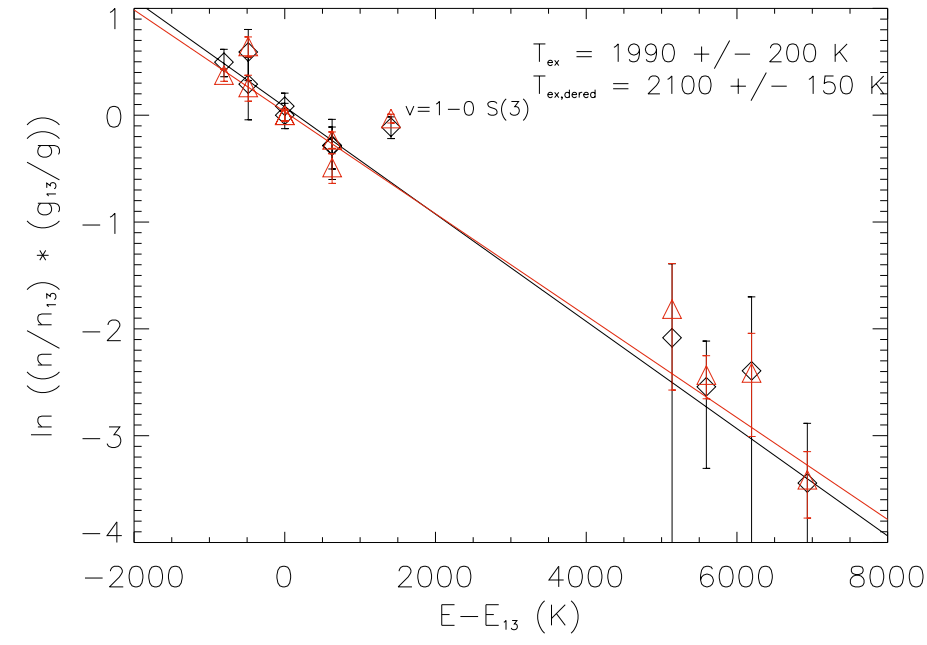

Fig. 9. Excitation diagram of the $\mathrm{H}_{2}$ emitting region southeast of $\mathrm{T}$ Tau $\mathrm{S}$ at $\left(0^{\prime \prime},-11^{\prime \prime} 15\right)$ (Fig. 4). The black diamonds $(\diamond)$ mark the measured data and the red triangles $(\triangle)$ mark the dereddened data $\left(A_{V}=7.2\right)$. The black line represents the best fit to the measured data, excluding the data point at $E-E_{1,3}=1409 \mathrm{~K}$ (the $v=1-0 \mathrm{~S}$ (3) line), see text. The red line is the best fit to the dereddened data. Note that the column densities of the $v=1-0 Q$-branch lines line up with the column densities of the $v=1-0 S$-branch lines in the left hand side of the figure.

to $A_{V} \sim 7.2$, which is the measured extinction in the emission region 2 (Fig. 6). Dereddening causes small changes in the column densities which shifts the fit towards higher temperatures. The temperature shift is well within the uncertainty of the fit to the uncorrected data, however.

The excitation temperature has been derived in all spatial pixels, producing a map of excitation temperature (Fig. 10). 

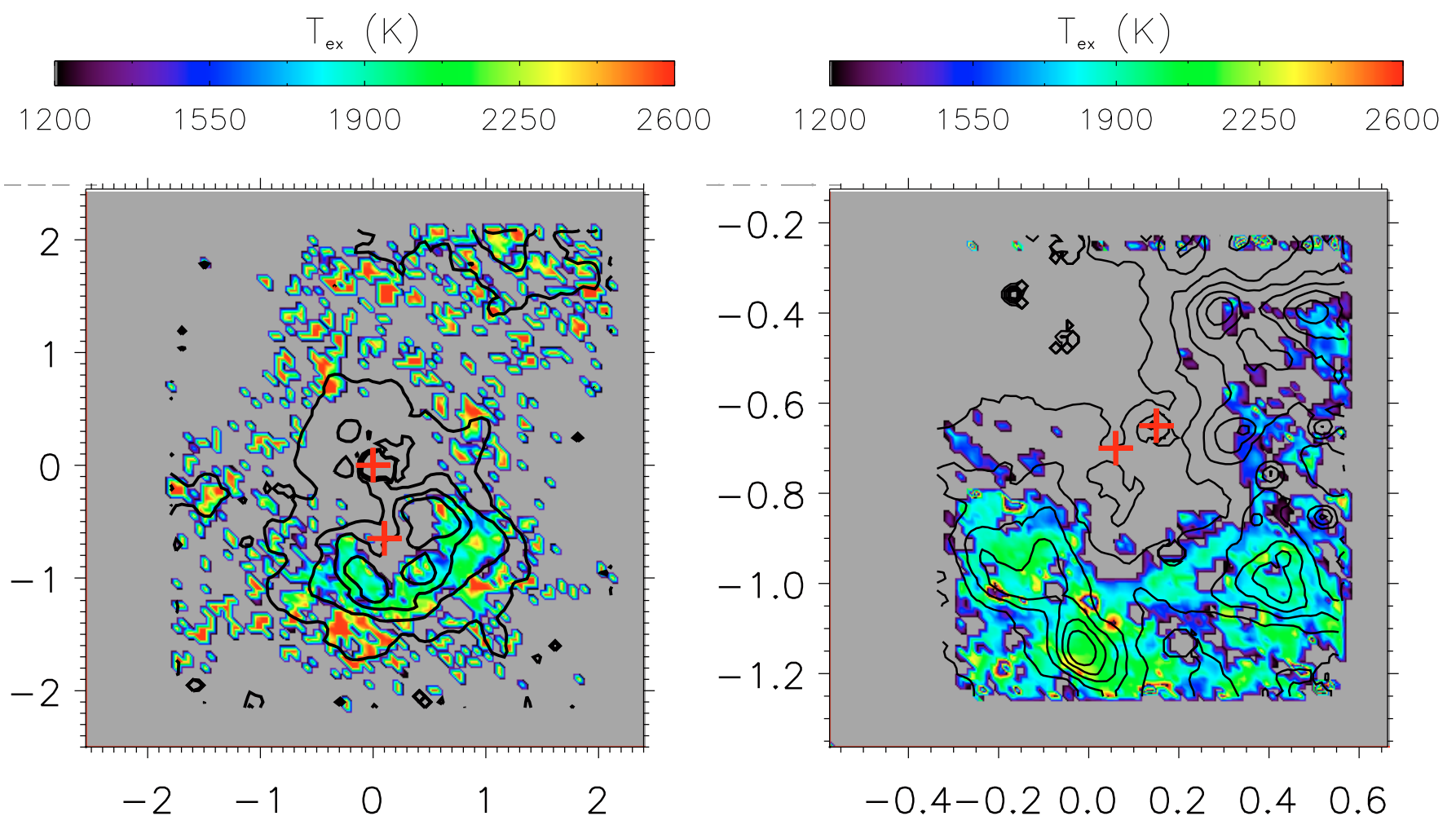

Fig. 10. Maps of excitation temperature. Left: 100 mas pixel scale, right: 25 mas pixel scale. The colourbars indicate the temperature in Kelvin. Only regions in which the $v=2-1 S$ (1) line is detected at a $2 \sigma$ level are displayed. Contours outline the $v=1-0 S(1)$ brightness.

Only lines which are detected at a $>2 \sigma$ level are included in the fit. This means that for the majority of spatial positions, only the $S$ (1) line from the $v=2-1$ band is included in the fit, since most of the other lines from this band are weak and are rejected in the fit. In regions where also the $v=2-1 S(1)$ line is not strong enough we do not perform a fit of the excitation temperature. The excitation temperature varies between $1200-2600 \mathrm{~K}$ and there is a trend showing increasing temperature with increasing distance from the stars. This trend affects the median temperature which is significantly higher in the large field of view $(2420 \mathrm{~K}$, Fig. 10 left) than in the small field of view (1400 K, Fig. 10 right). Both the median temperature and the temperature structure in the large field of view are consistent with the temperature map derived in Beck et al. (2008).

From Fig. 10, it is clear that the local peaks in $\mathrm{H}_{2} v=1-0$ $S$ (1) emission are associated with a large range of excitation temperatures. The excitation temperature in the emission peaks at $\left(0.35,-0{ }^{\prime} 65\right)$ and $\left(0.3,-0{ }^{\prime} 4\right)$ (labelled 4 and 5 in Fig. 3$)$ is approximately $1300 \mathrm{~K}$, while it is roughly $2100 \mathrm{~K}$ in the peak at $\left(-0 \prime^{\prime} 02,-1{ }^{\prime \prime} 15\right)$ (2 in Fig. 3). The cooler regions are generally associated with more extinction than the warmer regions, but although dereddening of the $\mathrm{H}_{2}$ lines would result in higher temperatures, it is not sufficient to explain the temperature differences. The difference in excitation temperature is real and indicates that the excitation conditions of molecular hydrogen are not the same throughout the region.

\subsubsection{Radial velocity}

The radial velocity of $\mathrm{H}_{2}$ emitting gas has been calculated using the $v=1-0 S$ (1) line at $2.12 \mu \mathrm{m}$. Although SINFONI only has a spectral resolution of $\sim 75 \mathrm{~km} \mathrm{~s}^{-1}$ in the $K$-band, it is possible to determine the peak position of the lines with much higher accuracy through line fitting. We have derived the radial velocity corresponding to every $\mathrm{H}_{2}$ emitting position by fitting a Gaussian profile to the unresolved line profiles on a pixel by pixel basis and the velocity is found from the fitted peak position, $\lambda_{0}$ (see Sect. 3.2). In our data, we do not find multiple peaks in the spectral profiles. If more than one kinematic component should be present in the line-of-sight, the multiple peaks in the spectrum are smoothed by the spectral profile of SINFONI to the point where the individual peaks are indistinguishable. The derived radial velocity effectively corresponds to the centroid velocity. The radial velocities have been corrected for the Earth's motion around the Sun at the time of observations. All velocities are quoted as local velocities with respect to the rest velocity of T Tau Sa of $+22.0 \mathrm{~km} \mathrm{~s}^{-1}$ (Duchêne et al. 2005). For reference, the heliocentric radial velocity of T Tau $\mathrm{Sb}$ is $+21.1 \mathrm{~km} \mathrm{~s}^{-1}$ (Duchêne et al. 2005), while the radial velocity of T Tau $\mathrm{N}$ is $+19.1 \mathrm{~km} \mathrm{~s}^{-1}$ (Hartmann et al. 1986). In regions of the 25 mas pixel scale image with strong signal and a high signal-to-noise ratio, the estimated uncertainty from the fit, $\Delta \lambda_{0}$, is on the order of $3-4 \mathrm{~km} \mathrm{~s}^{-1}$. In regions with weaker emission the uncertainty is larger and may be as large as $20 \mathrm{~km} \mathrm{~s}^{-1}$. For the 100 mas pixel size data, the typical uncertainty is $\sim 10 \mathrm{~km} \mathrm{~s}^{-1}$ in strong emission regions and up to $50 \mathrm{~km} \mathrm{~s}^{-1}$ in weak emission regions. The larger uncertainty in the 100 mas pixel size data is due to lower signal-to-noise ratio than in the 25 mas data.

The velocity field appears in Fig. 11. In the left hand side of Fig. 11, we see that the radial velocities in the immediate surroundings of $\mathrm{T}$ Tau $\mathrm{N}$ are $\sim-2 \mathrm{~km} \mathrm{~s}^{-1}$, that is, almost at rest in the rest frame of $\mathrm{T}$ Tau $\mathrm{N}$. The gas around $\mathrm{T}$ Tau $\mathrm{N}$ is associated with a nearly face-on circumstellar disk (Gustafsson et al. 2008). South of T Tau N, the velocity of the gas is $\sim-22 \mathrm{~km} \mathrm{~s}^{-1}$ and the velocity of the NW filament is $\sim-7 \mathrm{~km} \mathrm{~s}^{-1}$. The measured velocities are consistent with the velocities recorded in 


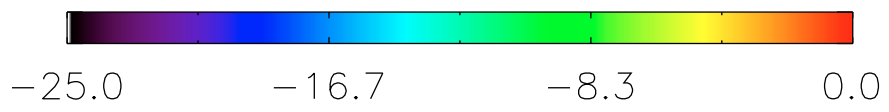

$$
\begin{array}{llll}
-25.0 & -16.7 & -8.3 & 0.0
\end{array}
$$
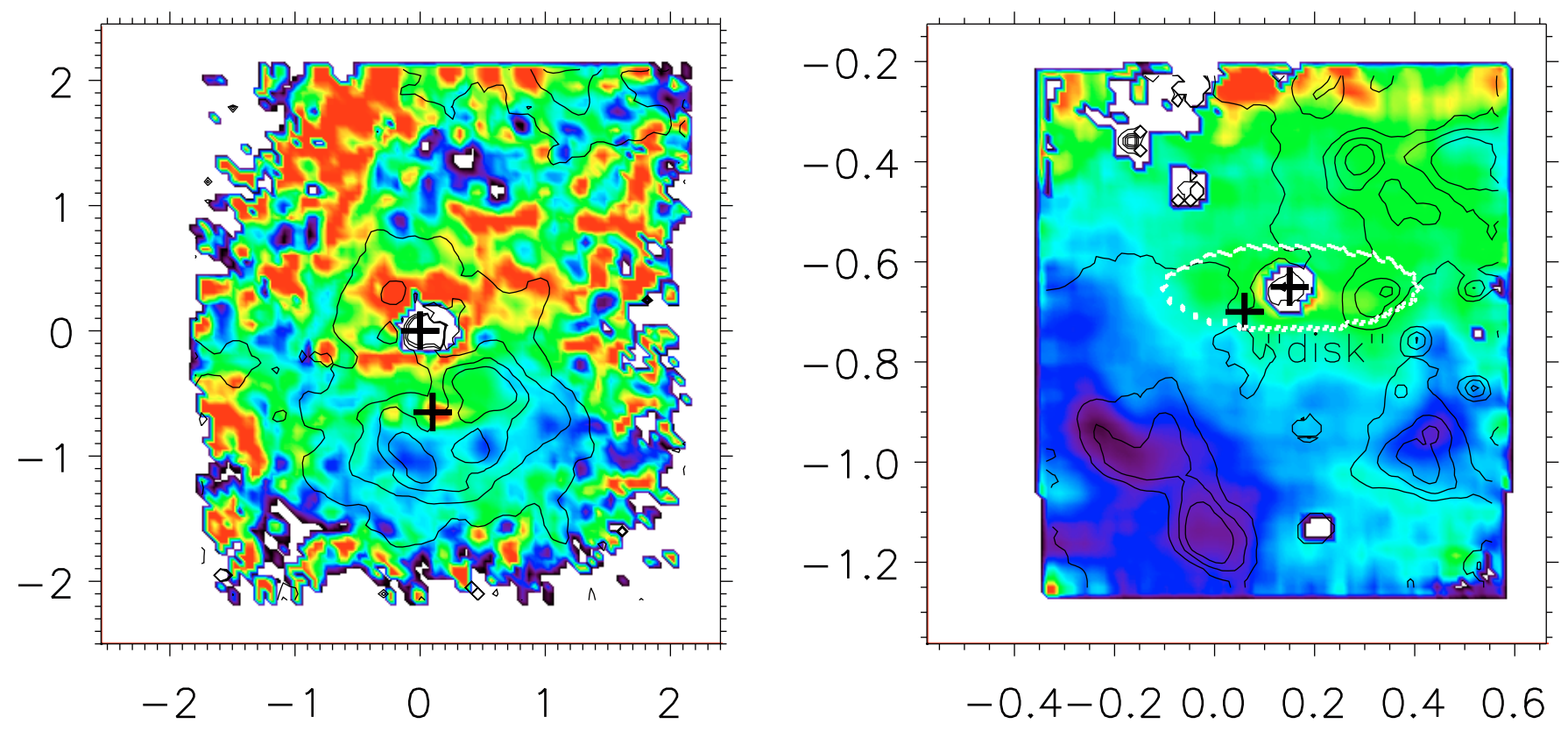

Fig. 11. Maps of radial velocity. Left: 100 mas pixel scale, right: 25 pixel scale mas. The colourbars indicate velocity in $\mathrm{km} \mathrm{s}^{-1}$ with respect to the rest velocity of T Tau Sa of $22.0 \mathrm{~km} \mathrm{~s}^{-1}$. Contours outline the $v=1-0 S(1)$ line brightness and only regions in which the $v=1-0 S(1)$ line is detected at a $3 \sigma$ level are displayed.

Herbst et al. (1997) and Duchêne et al. (2005), as well as the velocity gradient in the southeast-northwest direction detected in Herbst et al. (1997).

From the close-up (25 mas pixel scale data - right hand side in figure), we find that the $\mathrm{H}_{2}$ blobs southeast and southwest of $\mathrm{T}$ Tau S are $\sim 20 \mathrm{~km} \mathrm{~s}^{-1}$ blue-shifted with respect to the stars. There seems to be a velocity gradient with increasing velocity from the southeast to the northwest of T Tau S. The northwest corner is however still slightly blue-shifted with respect to the stars. The horseshoe-shaped emission region southeast of T Tau S (1a/1b in Fig. 3) shows two distinct velocity regions. The eastern most side (1a) has the highest velocity of $\sim-22 \mathrm{~km} \mathrm{~s}^{-1}$ while the western side (1b) shows significantly lower velocities of $\sim-19 \mathrm{~km} \mathrm{~s}^{-1}$. This again argues that the horseshoe-shaped emission feature is the result of two individual shocks, as suggested in Sect. 3.2.2 based on the distribution of the $v=2-1$ $S$ (1) emission. The radial velocities of the seven flows marked in Fig. 3 are listed in Table 2.

In the immediate vicinity of T Tau Sb (at $\left(0{ }^{\prime} 2,-0{ }^{\prime} 7\right)$, labelled "disk" in Fig. 11) there is an elongated feature with velocities close to the velocity of the stars. The uncertainties on the velocities of this feature are $\sim 10 \mathrm{~km} \mathrm{~s}^{-1}$, which are large compared to the span of velocities in the surroundings $\left(\sim 20 \mathrm{~km} \mathrm{~s}^{-1}\right)$. However, the feature shows up in each individual exposure with different nodding offsets and it is also clearly distinguishable in the 100 mas data (left hand side of Fig. 11). Thus, little doubt can remain that this feature is real and we speculate that it might be related to a rotating circumstellar or circumbinary disk within the T Tau S system.

The velocities measured here are consistent with the results from Duchêne et al. (2005), who found that the emission became increasingly blue-shifted moving away from the southern binary in the east-west direction until a blue-shift of $10 \mathrm{~km} \mathrm{~s}^{-1}$ was reached $\sim 0$ ' $4-0$ '! 5 away on either side of the stars. The velocity map is also consistent with that of Beck et al. (2008).

\subsubsection{Proper motions}

Recently, Herbst et al. (2007) published high spatial resolution images of $\mathrm{H}_{2}$ in the T Tau system. Their data were obtained with NACO on the ESO-VLT on the nights of December 14-15 2002. By a direct comparison where we plot the data of Herbst et al. (2007) together with our data (Fig. 12) we can see how the $\mathrm{H}_{2}$ emission has evolved in the central region of T Tau on a timescale of $\sim 2$ years. The data are aligned on $\mathrm{T} \mathrm{Tau} \mathrm{Sa,} \mathrm{which}$ is the most massive star in the triple system (Köhler 2008).

From the large field of view, it seems that the NW emission knot, of which only the southern tip is visible in our data, has remained in the same position during the time-span of 2 years. The outline of the main emission region around T Tau $\mathrm{S}$ is also roughly the same in the two epochs, although the contours suggest that the southern part has expanded. No proper motions are detectable in these data for the $\mathrm{H}_{2}$ flows NW and W of T Tau S labelled C1 and C2 in Herbst et al. (2007) (flow called bow and nr 5+6 in Fig. 3). The proper motions of the flows south of T Tau S, labelled C3 and C4 in Herbst et al. (2007) are discussed below.

A more detailed picture is found in the smaller field. The strongly emitting peak or complex of peaks to the northwest of T Tau S (4, 5, 6 in Fig. 3, C2 in Herbst et al. 2007) does not seem to have changed position. In contrast, the southwestern peak (3 in Fig. 3, C3 in Herbst et al. 2007), which resembles a bow shock originating in one of the T Tau S stellar components, has moved away from the stars by $\sim 0$ ' 09 , corresponding to an 

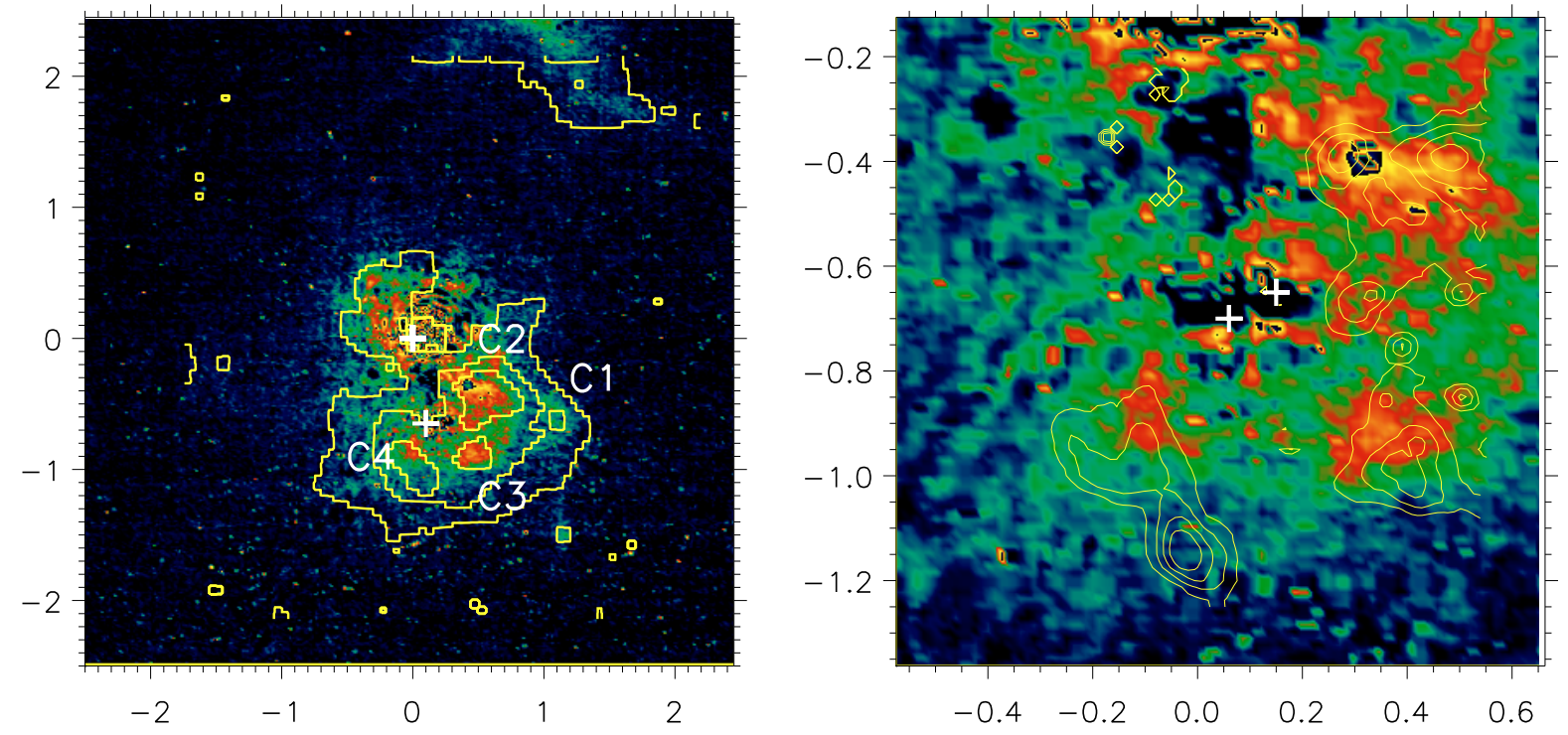

Fig. 12. $\mathrm{H}_{2} v=1-0 S(1)$ emission from December 2002 (colours, Herbst et al. 2007) and November 2004 (contours, our data). The data are aligned on T Tau Sa both in the large field of view image (left) and in the small field of view (right). The labeling of the flows of Herbst et al. (2007) is displayed in the large field of view for reference.

average velocity of $\sim 32 \mathrm{~km} \mathrm{~s}^{-1}$. Using the radial velocity of $\sim 17 \mathrm{~km} \mathrm{~s}^{-1}$ with respect to $\mathrm{T} \mathrm{Tau} \mathrm{Sa} / \mathrm{Sb}$ found in this bow shock (Fig. 11) we derive a $3 \mathrm{D}$ velocity of $\sim 36 \mathrm{~km} \mathrm{~s}^{-1}$ and an inclination of the flow to the line of sight of $\sim 60^{\circ}$. The direction of the proper motion indicates that this bow shock comes from $\mathrm{T} \mathrm{Tau} \mathrm{Sb.}$

The peak which appeared southeast at $(-0.05,-0.95)$ in 2002 (C4 in Herbst et al. 2007) has clearly developed, but it is unclear with which blob in the 2004 data the 2002 blob should be associated. It could have developed into either of the two southeastern blobs at $\left(0{ }^{\prime} 05,-1{ }^{\prime \prime} .2\right)$ and $\left(-0{ }^{\prime} 1,-1{ }^{\prime \prime} .05\right)(1 \mathrm{a} / 1 \mathrm{~b}$ and 2 in Fig. 3). As mentioned above, the horseshoe-shaped flow at $\left.\left(-0{ }^{\prime} 1,-1\right)^{\prime \prime} 05\right)$ is most likely made up of two distinct flows. Thus, the 2002 southeast blob could have developed into any of the three flows in 2004. Judging from the morphology and the implied direction of the proper motions, we consider it most likely that the 2002 blob has developed into the eastern flow of the horseshoe (1a). This flow would then originate in T Tau Sa and from the proper motion of 0 .' 15 we derive a space velocity of $\sim 53 \mathrm{~km} \mathrm{~s}^{-1}$. Together with the radial velocity of $\sim 22 \mathrm{~km} \mathrm{~s}^{-1}$ with respect to $\mathrm{T}$ Tau Sa the $3 \mathrm{D}$ velocity is then $\sim 57 \mathrm{~km} \mathrm{~s}^{-1}$ and the inclination of the flow is $\sim 70^{\circ}$.

\subsection{Lines in J-band}

Only two extended emission lines were detected in the $J$-band, namely $\mathrm{Pa} \beta$ at $1.282 \mu \mathrm{m}$ and [Fe II] at $1.257 \mu \mathrm{m}$. The fact that $\operatorname{Pa} \beta$ is observed to be spatially extended is most likely due to scattering of light from the stars - mainly T Tau N - and not local emission. Further evidence favouring this conclusion is given by the fact that the $\mathrm{Pa} \beta$ line to continuum ratio is more or less constant over the whole field of view. The extent of the continuum emission and thus $\mathrm{Pa} \beta$ can be seen in Fig. 1 .

The [Fe II] emission shows a different spatial distribution (Fig. 13) to that of $\mathrm{Pa} \beta$ with a region of very strong emission in the northern part of the field between T Tau S and T Tau N, extending to the west. [Fe II] emission must therefore be emitted

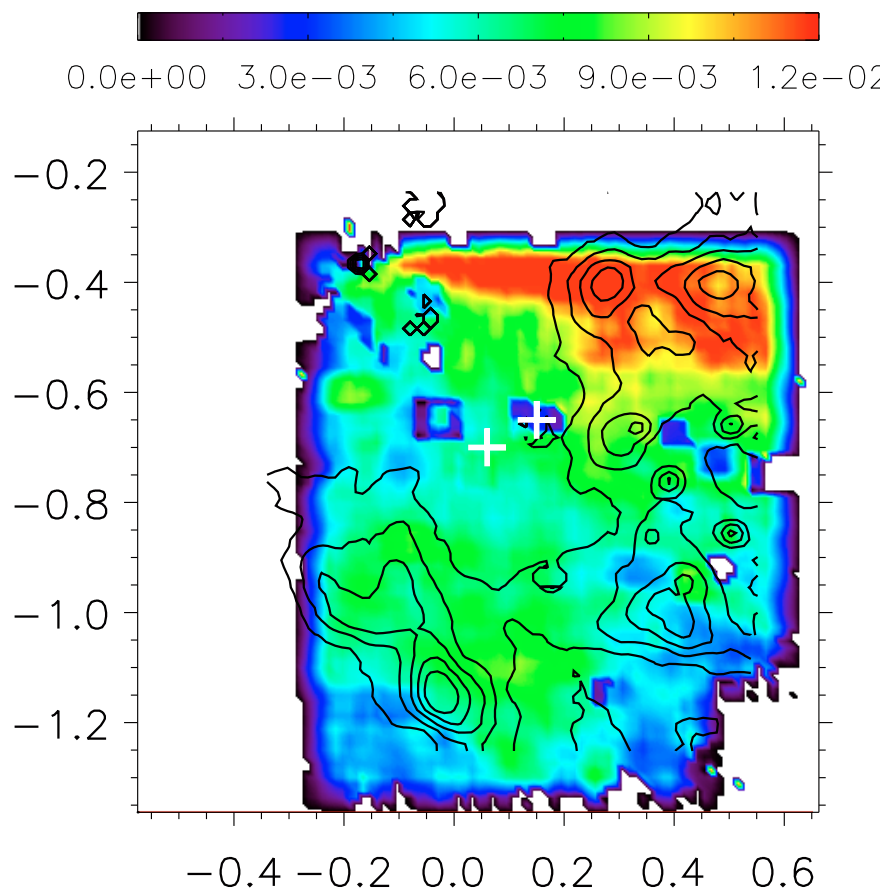

Fig. 13. Small field of view. [Fe II] emission at $1.257 \mu \mathrm{m}$ in $\mathrm{erg} \mathrm{s}^{-1} \mathrm{~cm}^{-2} \mathrm{sr}^{-1}$ (colours) and $\mathrm{H}_{2} v=1-0 \mathrm{~S}$ (1) emission (contours).

locally in the ambient gas. To the southeast of $\mathrm{T}$ Tau $\mathrm{S}$ there is a strong [Fe II] emission region as well. It is also clear that [Fe II] and $\mathrm{H}_{2}$ emission are not spatially coincident. In the region between T Tau $\mathrm{N}$ and $\mathrm{T}$ Tau S both the $\mathrm{H}_{2}$ and the [Fe II] emission are strong, whereas we detect very little [Fe II] emission from the $\mathrm{H}_{2}$ flow to the southwest of T Tau S (flow nr 3). To the southeast of T Tau S both $\mathrm{H}_{2}$ and $\mathrm{Fe}$ emission is detected but the peak of the [Fe II] emission is spatially offset from that of the $\mathrm{H}_{2}$ and is found closer to the stars. The different spatial distributions of 
Table 3. Best fit 1D shock models to the seven flows in Fig. 3.

\begin{tabular}{cccccccccc}
\hline \hline Region & Type & $n \mathrm{H}\left(\mathrm{cm}^{-3}\right)$ & $v\left(\mathrm{~km} \mathrm{~s}^{-1}\right)$ & $B(\mathrm{mG})$ & $\mathrm{o} / \mathrm{p}$ & width $(\mathrm{AU})$ & $t_{\text {cool }}(\mathrm{yr})$ & $\begin{array}{c}I_{v=1-0 S(1)} \\
\left(10^{-2} \mathrm{erg} \mathrm{s}^{-1} \mathrm{~cm}^{-2} \mathrm{sr}^{-1}\right)\end{array}$ & $\begin{array}{c}I_{v=2-1 S(1)} \\
\left(10^{-2} \mathrm{erg} \mathrm{s}^{-1} \mathrm{~cm}^{-2} \mathrm{sr}^{-1}\right)\end{array}$ \\
\hline $1 \mathrm{a}$ & $\mathrm{c}$ & $5 \times 10^{5}$ & 21 & 0.35 & 3 & 10 & 3.7 & 1.27 & 0.104 \\
$1 \mathrm{~b}$ & $\mathrm{c}$ & $5 \times 10^{5}$ & 21 & 0.35 & 3 & 10 & 3.7 & 1.27 & 0.104 \\
2 & $\mathrm{c}$ & $5 \times 10^{5}$ & 24 & 0.70 & 3 & 19 & 5.7 & 1.64 & 0.101 \\
3 & $\mathrm{c}$ & $1 \times 10^{6}$ & 17 & 0.50 & 3 & 5 & 2.6 & 1.33 & 0.094 \\
4 & $\mathrm{c}$ & $5 \times 10^{5}$ & 21 & 0.35 & 3 & 10 & 3.7 & 1.27 & 0.104 \\
5 & $\mathrm{c}$ & $5 \times 10^{5}$ & 23 & 0.70 & 3 & 19 & 6.1 & 1.34 & 0.069 \\
6 & $\mathrm{c}$ & $5 \times 10^{5}$ & 21 & 0.35 & 3 & 10 & 3.7 & 1.27 & 0.104 \\
\hline
\end{tabular}

$\mathrm{H}_{2}$ and $\mathrm{Fe}$ emission is to be expected since [Fe II] emission is mainly produced in J-type shocks where $\mathrm{H}_{2}$ is dissociated and the $\mathrm{H}_{2}$ emission is consequently low.

\section{Discussion}

We now use the measurements of the $\mathrm{H}_{2}$ emission to model the physical conditions in the environment of $\mathrm{T}$ Tau. $\mathrm{H}_{2}$ emission offset from the central source is usually associated with shocks produced by outflows and the excitation pattern of $\mathrm{H}_{2}$ is very sensitive to the shock conditions. We seek to reproduce the $\mathrm{H}_{2}$ brightness of the seven emission regions given in Table 2 with state-of-the-art planar shock models in order to estimate the underlying shock parameters.

First, we note that the size of the observed shocks constrains the density of the pre-shock gas. In a $n_{H} \sim 10^{5} \mathrm{~cm}^{-3}$ medium, the shock width is $\sim 100 \mathrm{AU}$ (see Fig. 8 in Kristensen et al. 2007), that is, much larger than the size of the observed shocks. On the other hand, in a $n_{H} \sim 10^{7} \mathrm{~cm}^{-3}$ medium the shock width is a few $\mathrm{AU}$, which is very narrow compared to what we observe even if projection effects are considered. Thus, the pre-shock densities are most likely to be found in the $5 \times 10^{5}-5 \times 10^{6} \mathrm{~cm}^{-3}$ range.

We use the grid of planar C-shocks described in Kristensen et al. (2007) and a $\chi^{2}$ analysis to find the shock that best matches the observed line emission of the $12 \mathrm{H}_{2}$ lines. The grid in Kristensen et al. (2007) was calculated using the shock code described in Flower \& Pineau des Forêts (2003) and references therein, in which a large chemical reaction network with 1065 processes involving 136 species is included. The range of input parameters in the shock grid is as follows:

- pre-shock density, $n_{H}: 10^{4}, 5 \times 10^{4}, 10^{5}, 5 \times 10^{5}, 10^{6}, 5 \times 10^{6}$, $10^{7} \mathrm{~cm}^{-3}$

- shock velocity: $10-50 \mathrm{~km} \mathrm{~s}^{-1}$, step size of $1 \mathrm{~km} \mathrm{~s}^{-1}$;

- transverse magnetic flux density, $B=b \times n_{H}^{1 / 2} \mu \mathrm{G}$ with the scaling factor $b=0.5-10$, step size of 0.5 ;

- initial ortho/para ratio: 3.0 .

For every model in the grid and all seven emission regions we calculate $\chi^{2}=\sum \frac{X_{\mathrm{obs}}-X_{\text {model }}}{\sigma_{\text {obs }}}$, where $X_{\mathrm{obs}}$ and $X_{\text {model }}$ refer to the observed and modelled quantities, respectively. $\sigma_{\text {obs }}$ refers to the uncertainty associated with the observed parameter. We include the brightness of the $12 \mathrm{H}_{2}$ lines and the size of the emitting regions in the $\chi^{2}$ evaluation. The shock parameters of the best fit models that minimizes the $\chi^{2}$ for each emitting region are listed in Table 3 along with the shock width, the cooling time of the excited hydrogen and the modelled line emission of the $v=1-0 S(1)$ and the $v=2-1 S(1)$ lines. We have also tested if the observed line brightness can be achieved in non-magnetic $J$ shocks calculated with the shock code described in Flower et al. (2003). That is not the case.
The modelling of the flows in the vicinity of $\mathrm{T}$ Tau shows that the ambient material is very dense with a pre-shock density of $\sim 5 \times 10^{5} \mathrm{~cm}^{-3}$. All flows are consistent with being excited by a shock wave moving at a speed of $17-24 \mathrm{~km} \mathrm{~s}^{-1}$ in a $B=0.35-0.70 \mathrm{mG}$ medium. These shock velocities are considerable lower than the $3 \mathrm{D}$ velocities of flow $1 \mathrm{a}\left(57 \mathrm{~km} \mathrm{~s}^{-1}\right)$ and 3 $\left(36 \mathrm{~km} \mathrm{~s}^{-1}\right)$ derived in Sect. 3.2.5 from the radial velocities and proper motions. One way to reconcile these seemingly contradictory facts is if the shocked medium has been accelerated prior to the arrival of the shock wave. This could have been done by a previous passing shock wave that simultaneously would have compressed the gas. If this scenario is true, the velocity of the impinging outflow is higher than the modelled shock velocity and at least as high as the 3D velocity of the flows.

The complex outflow pattern with many arcs and filaments within $10^{\prime \prime}$ of the stars (Herbst et al. 1997) is also evidence of past episodic flows. Further support of the idea that shock waves have previously crossed the region is found in the short cooling times of the modelled shocks of a few years. The cooling time of a shock is closely associated with the cooling distance and is therefore largely set by the observed size of the emission region. Given the tight constraint the observed shock width puts on the cooling time, it is certain that the flows we see have not been detectable for more than a few years. Therefore, a succesive generation of shocks seems necessary in order to explain that the presence of strong $\mathrm{H}_{2}$ emission in the inner $1^{\prime \prime}$ of T Tau $\mathrm{S}$ has been observed for more than a decade (e.g. Herbst et al. 1996). With the recent high spatial resolution data, it has for the first time become feasible to trace individual flows, and with future follow up observations, these ideas can be fully tested.

The modelling of the flows in T Tau shows that flow 1a and $1 \mathrm{~b}$ can both - even though treated independently - arise from a $\mathrm{C}$-shock with the same parameters. Perhaps they should not be attributed to two different shock waves afterall. 3D modelling of shocks shows that a bow shock can create a horseshoe shaped emission morphology like that of $1 \mathrm{a} / 1 \mathrm{~b}$ if the direction of the magnetic field is inclined with respect to the propagation axis of the bow shock and the shock is moving at an angle to the lineof-sight of $\sim 40^{\circ}-50^{\circ}$ (Fig. A.3 in Gustafsson et al. 2009). The radial velocity map of a shock with this geometry (Fig. 10 in Gustafsson et al. 2009) is also consistent with our observations (Fig. 11). One side of the horseshoe displays significantly higher blueshifted velocities than the other side.

The seven flows in the vicinity of $\mathrm{T}$ Tau $\mathrm{S}$ have all been successfully modelled with C-type shocks. In C-shocks the production of [Fe II] emission is very low and not sufficient to explain the observed [Fe II] brightness. Instead, the [Fe II] brightness indicates that J-type shocks in which [Fe II] emission is more readily produced surround the $C$-shocks. In the simplest possible geometry, where a bow shock is seen edge-on, $J$-shocks are usually found on the outside of C-shocks. At other inclinations the 

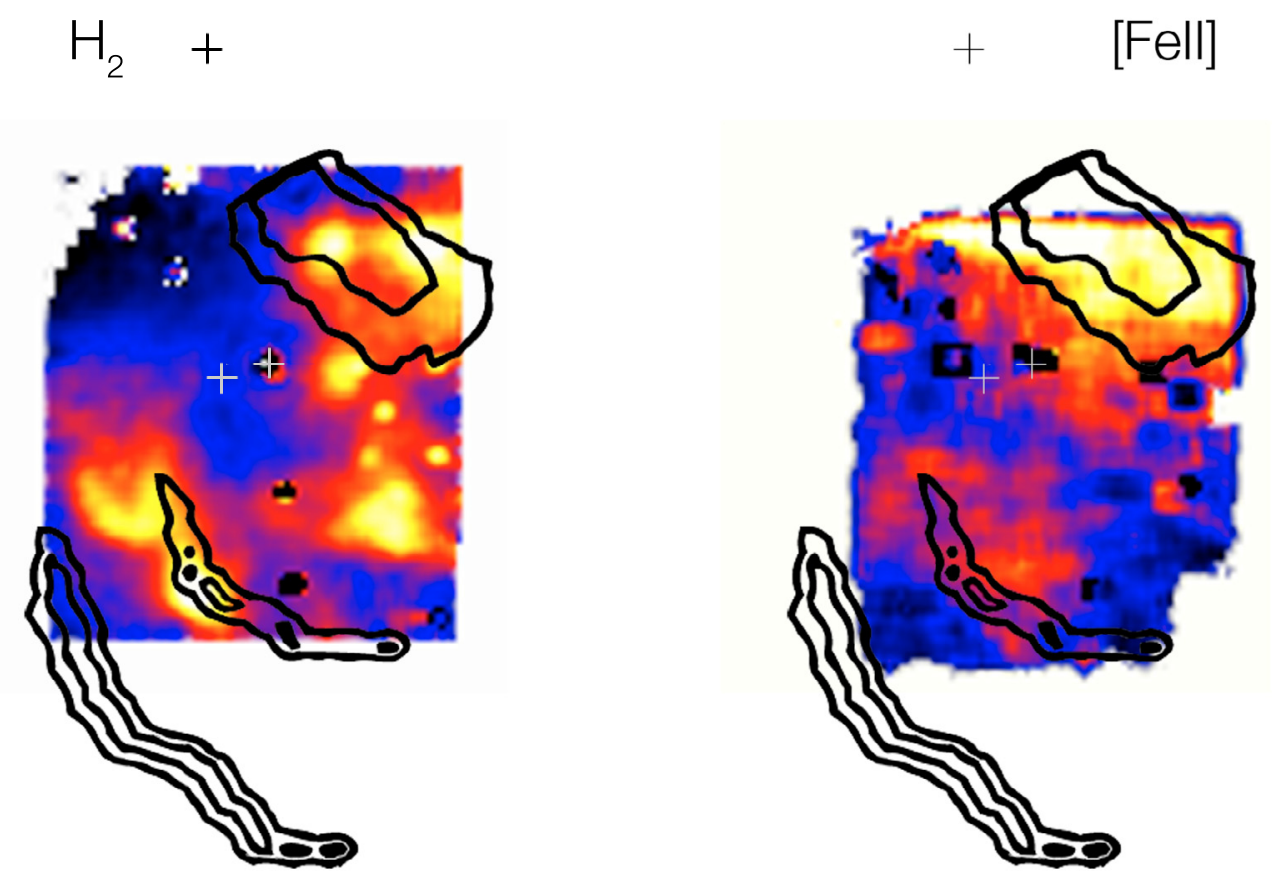

Fig. 14. Overlay of the UV fluorescent $\mathrm{H}_{2}$ emission (contours) from Saucedo et al. (2003) on the $\mathrm{H}_{2} 2.12 \mu$ m map (left) and the [Fe II] map (right). The positions of the three stars are indicated with crosses.

Fe emission from $J$-shocks may appear on the inside of the $\mathrm{H}_{2}$ emission produced by $C$-shocks (Gustafsson et al. 2009). This is indeed what we see to the southeast of $\mathrm{T}$ Tau $\mathrm{S}$.

Saucedo et al. (2003) detected UV fluorescent $\mathrm{H}_{2}$ emission in two arcs south of T Tau S close to the $2.12 \mu \mathrm{m}$ flows $(1 \mathrm{a}, 1 \mathrm{~b}, 2)$ and in a lobe SW of T Tau N coincident with the flows nr 5 and 6 here (Fig. 14). There is a considerable spatial overlap between flourescent $\mathrm{H}_{2}$ and [Fe II] emission (Fig. 14 right) and it is very plausible that the J-shocks associated with the [Fe II] emission powers the flourescence as suggested in Saucedo et al. (2003). It is interesting that the northern lobe of fluorescent $\mathrm{H}_{2}$ emission is spatially coincident with our flows nr 5 and 6 where little or no emission in the $\mathrm{H}_{2} v=2-1 S$ (1) line was detected. This led us to rule out fluorescence as the excitation mechanism of the nearinfrared $\mathrm{H}_{2}$ emission. From the distribution of the near-infrared $\mathrm{H}_{2}$ emission, the $\mathrm{UV}_{2}$ fluorescence and the [Fe II] emission, we conclude that the near-infrared $\mathrm{H}_{2}$ emission is dominated by excitation in $\mathrm{C}$-shocks. Fluorescence also contribute to the population of the $\mathrm{H}_{2}$ levels, but since it is powered by J-shocks the associated excitation is overwhelmed by the much stronger excitation from the $\mathrm{C}$-shocks.

\subsection{The origin of the flows}

There are two clear outflow systems arising from the three stars in the T Tau system, but it has proven difficult to settle to which star each outflow should be assigned. There is an east-west jet ending in the Herbig-Haro object HH 155 (Bührke et al. 1986) which was assigned to T Tau N by Böhm \& Solf (1994) and Solf \& Böhm (1999) on the basis of position-velocity diagrams from long-slit spectra. In contrast, near-infrared observations of $\mathrm{H}_{2}$ led Herbst et al. (1996) to associate T Tau S with the east-west flow. In Herbst et al. (2007) the morphology of the $\mathrm{H}_{2}$ flows in the vicinity of $\mathrm{T}$ Tau $\mathrm{S}$ was interpreted as a bow shock (C1 in Herbst et al. (2007), named "bow" in Fig. 3) coming from
T Tau S with bright, oblique shocks lining the flow channel (C2 and C3 in Herbst et al. (2007), flows number 3, 5, and 6 here). This would unambiguously identify T Tau S as the source of the east-west flow. This interpretation is however challenged by the proper motions of the $\mathrm{H}_{2}$ filaments derived in this paper. The proper motions show that the flow 3/C3 is moving in the southwest direction away from $\mathrm{T}$ Tau $\mathrm{S}$ whereas the flows $5+6 / \mathrm{C} 2$ and bow/C1 have not moved in the intermediate time period. It is difficult to see how such a flow pattern could arise in a single bow shock with limb brightened shocks in the wings. It is therefore more likely that the 3/C3 flow constitutes an independent outflow (the "missing" third outflow from the three stars?) and should not be associated with the $\mathrm{C} 1$ and $5+6 / \mathrm{C} 2$ flows. The proper motion shows that outflow 3 is coming from the southern binary with a preference for $\mathrm{T} \mathrm{Tau} \mathrm{Sb}$. However, given the small separation of the binary, $\mathrm{T}$ Tau Sa cannot be ruled out as the source.

From the geometry of the outflows and the radial velocity we argue that the flows $1 \mathrm{a} / 1 \mathrm{~b}$ and 2 are the blue-shifted counterparts to the more red-shifted NW flow (Herbst et al. 1997). The flows $1 \mathrm{a} / 1 \mathrm{~b}$ and 2 are thus part of the southeast-northwest outflow called C-D in Böhm \& Solf (1994). The proper motions pin-point the origin of the $1 \mathrm{a} / 1 \mathrm{~b}$ flow and thus the southeastnorthwest outflow to the T Tau S binary. Radio emission at $6 \mathrm{~cm}$ in the southeast-northwest direction has also been found centered on T Tau S (Ray et al. 1997) and recently van Boekel et al. (2009) found bright [NeII] emission centered on T Tau S and extending to the NW filament. All this is further evidence favouring $\mathrm{T}$ Tau $\mathrm{S}$ as the driving source of the southeast-northwest outflow. However, as with the flow $\mathrm{nr} 3$, there is an ambiguity between $\mathrm{T}$ Tau Sa and $\mathrm{Sb}$.

Loinard et al. (2007a) identified the radio source south of $\mathrm{T}$ Tau $\mathrm{N}$ with $\mathrm{T}$ Tau $\mathrm{Sb}$ and detected extended radio emission at $3.6 \mathrm{~cm}$ from this source at position angle $(\mathrm{PA})+30^{\circ}-70^{\circ}$ $\left(\right.$ or $\left.+210^{\circ}-250^{\circ}\right)$. The radio emission is presumably produced by the interaction of a stellar wind with circumstellar material. 
The PA of flow 3 is $220^{\circ}$ with respect to T Tau Sb and is remarkably close to the PA of the radio emission. We argue that the flow 3 originates in $\mathrm{T} \mathrm{Tau} \mathrm{Sb}$ and is one of the shocks produced when the wind from the star impinges on the ambient gas.

Based on mid-infrared interferometry Ratzka et al. (2009) modelled the inclination of the disk around T Tau Sa to be $\sim 72^{\circ}$ compared to $i=\sim 35^{\circ}$ for the disk around $\mathrm{T}$ Tau Sb. The inclination of the disk of T Tau Sa fits very well with the inclination of the southeast-northwest outflow of $79^{\circ}$ (Solf \& Böhm 1999). We have used the proper motions and radial velocities to estimate the inclination of flow $1 \mathrm{a}$. The inclination, $i=\arctan \left(v_{\tan } / v_{\mathrm{rad}}\right) \sim 70^{\circ}$ is consistent with the inclination of the southeast-northwest outflow. This all seems to favour $\mathrm{T}$ Tau $\mathrm{Sa}$ as the source of the southeast-northwest flow and $\mathrm{T} \mathrm{Tau} \mathrm{Sb}$ as the source of the "new" southwest outflow. The inclination of flow 3 of $\sim 60^{\circ}$ is larger than the inferred inclination of the disk around $\mathrm{T}$ Tau $\mathrm{Sb}$ of $35^{\circ}$ (Ratzka et al. 2009) but since both results are relatively uncertain the values are not irreconcilable.

Since the orbital period of the southern binary is only 2128 years (Duchêne et al. 2006; Köhler 2008) the position of the stellar components was quite different when the exciting jets were launched than at the time of observation. If we assume that the velocity of the jets equals the derived space velocity $-32 \mathrm{~km} \mathrm{~s}^{-1}$ and $53 \mathrm{~km} \mathrm{~s}^{-1}$ of flow 3 and 1a, respectively (Sect. 3.2.5) - the exciting jets from $\mathrm{T}$ Tau $\mathrm{Sa}$ and $\mathrm{T} \mathrm{Tau} \mathrm{Sb}$ should have been launched close to the periastron passage, namely between 1995 and 1999. In that period the position angle was $\sim 140-240^{\circ}$ (Köhler 2008). The star-jet association above is fully consistent with the stellar positions at the time of launch. The extended structure $1 \mathrm{a} / 1 \mathrm{~b} / 2$, consisting of short-lived emission regions, originating from a sequence of outflows, might be the result of precession of the jet from $\mathrm{T}$ Tau Sa due to the orbital motion of $\mathrm{T}$ Tau $\mathrm{Sb}$. Thus, the $\mathrm{H}_{2}$ emission regions might bear witness to the short orbital period of the binary.

If the southern binary drives the southeast-northwest and the southwest outflow, $\mathrm{T}$ Tau $\mathrm{N}$ must be the driving source of the east-west flow, which in our data are represented by flow $5+6$ and the flow called "bow". That is further supported by the presence of extended radio emission at $2 \mathrm{~cm}$ from $\mathrm{T}$ Tau $\mathrm{N}$ at position angle $60^{\circ}-65^{\circ}$ or $240^{\circ}-245^{\circ}$ (Loinard et al. 2007a). The angle of the radio outflow is consistent with the PA from $\mathrm{T}$ Tau $\mathrm{N}$ to "bow" of $\sim 235^{\circ}$. Furthermore, the low (unmeasurable) tangential velocities in the flow $5+6$ and "bow" indicate that the eastwest flow is moving at a low inclination to the line of sight. The inclination of $\mathrm{T}$ Tau $\mathrm{N}$ is $19^{\circ}$ (Herbst et al. 1997) and the inclination of HH 155 which is believed to be the working surface of the east-west jet is $23^{\circ}$ (Eislöffel \& Mundt 1998). Our proposed solution to the enigma of the outflows in T Tauri is summarized in Table 4 . The proposed association of stars and flow implies that all three disks are misaligned and that three different stars are driving the flows in the immediate vicinity of T Tau S. T Tau Sa drives flow 1a/1b and 2, T Tau Sb drives flow nr 3 and T Tau N drives flow $\mathrm{nr} 5$ and 6 . With this association it is easily explained why all flows in a $270^{\circ}$ angle around $\mathrm{T}$ Tau $\mathrm{S}$ are blueshifted. The misalignment of the three disks in T Tauri is quite puzzling. For a detailed discussion of possible formation scenarios we refer to Ratzka et al. (2009).

\subsection{The nature of the obscuring material}

From our extinction maps (Fig. 6) it is evident that the extinction is high in the immediate surroundings of $\mathrm{T}$ Tau $\mathrm{S}$ and in the area between $\mathrm{T}$ Tau $\mathrm{N}$ and $\mathrm{T}$ Tau S. As mentioned previously (Fig. 6 left) the obscuring material between the north and
Table 4. Outflows and associated sources in T Tauri.

\begin{tabular}{ccccc}
\hline \hline Star & Outflow & $\mathrm{H}_{2}$ flow & $\left.\mathrm{PA}^{\circ}\right)$ & Inclination $\left(^{\circ}\right)$ \\
\hline $\mathrm{N}$ & $\mathrm{E}-\mathrm{W}$ & 5,6, "bow" & $235-245^{a},{ }^{b}$ & $19-23^{c},{ }^{d}$ \\
$\mathrm{Sa}$ & $\mathrm{SE}-\mathrm{NW}$ & $1 \mathrm{a} / 1 \mathrm{~b}, 2$ & $100-190^{a}$ & $79^{e}$ \\
$\mathrm{Sb}$ & $\mathrm{SW}$ & 3 & $210-250^{a},{ }^{b}$ & $35-60^{a},{ }^{g}$ \\
\hline
\end{tabular}

Notes. ${ }^{(a)}$ This work; ${ }^{(b)}$ Loinard et al. (2007a); ${ }^{(c)}$ Herbst et al. (1997); ${ }^{(d)}$ Eislöffel \& Mundt (1998); ${ }^{(e)}$ Solf \& Böhm (1999); ${ }^{(g)}$ Ratzka et al. (2009) .

south components is seen to be part of a filament that continues to the east of T Tau. South of T Tau S, the extinction is lower by $A_{V} \sim 15$. The extinction close to $\mathrm{T}$ Tau $\mathrm{N}$ cannot be assessed due to the large amount of scattered light which swamps the $\mathrm{H}_{2}$ lines. The extinction of $A_{V} \sim 20$ close to T Tau $\mathrm{S}$ is consistent with what previous authors find towards $\mathrm{T}$ Tau $\mathrm{S}\left(A_{V}=17.4\right.$, van den Ancker et al. (1999); $A_{V}=5-27$, Beck et al. 2004). The region of high extinction northeast of $\mathrm{T}$ Tau $\mathrm{S}$ may be associated with the $0 . .5 \times 0 . ' 64$ structure of obscuring material observed by Walter et al. (2003). From our map, it is clear that the northeast structure does not resemble a simple edge-on disk around T Tau Sa as suggested by Walter et al. (2003) or a circumbinary disk around both T Tau S components (Duchêne et al. 2005). Instead, we suggest that the obscuration is due to a combination of a circumbinary disk around $\mathrm{T}$ Tau $\mathrm{S}$, the circumstellar disk around T Tau N (Gustafsson et al. 2008) and a long (>2" in the east-west direction) dust filament between T Tau N and T Tau S. A circumstellar disk around T Tau Sa may also be present in order to explain the non-detection of this star at wavelengths shortward of $1.6 \mu \mathrm{m}$. The dust filament may be a result of the star-star interaction or it may be a foreground object.

\section{Summary}

We have presented new high spatial resolution observations of $\mathrm{T}$ Tau in the near-infrared. The integral field spectroscopic data obtained with SINFONI enables us to trace the emission produced in shocks from outflows in $12 \mathrm{H}_{2}$ rovibrational emission lines as well as the [Fe II] line at $1.257 \mu \mathrm{m}$.

$\mathrm{H}_{2}$ is detected everywhere within $2^{\prime \prime}$ of T Tau N, with the strongest emission concentrated around the southern binary $\mathrm{T}$ Tau S. A number of the strongly emitting regions have a bow shock morphology. We have not detected any $\mathrm{H}_{2}$ emission in the stellar components.

We have used four $\mathrm{H}_{2}$ lines - two pairs with the same upper energy level - to analyse the extinction around $\mathrm{T}$ Tau. The absolute values of $\mathrm{A}_{V}$ are highly uncertain and relies on high accuracy of flux determination in the lines involved. The variations in $\mathrm{A}_{V}$ are however uncorrelated with brightness and are believed to be real. The extinction is very high around T Tau $S$ and in the region between $\mathrm{T}$ Tau $\mathrm{S}$ and $\mathrm{N}$. The obscuring material seems to be part of a filament that stretches to the east of T Tau.

The $v=1-0 S(1) / v=2-1 S(1)$ line ratio is highly variable ranging from $\sim 8-20$. The high values are incompatible with excitation by UV fluorescence and indicates that all - or at least most - of the detected $\mathrm{H}_{2}$ emission arises in shocks.

We have derived the map of excitation temperature using all $\mathrm{H}_{2}$ lines which are detected at a $>2 \sigma$ level. The excitation temperature varies between $1200 \mathrm{~K}$ and $2600 \mathrm{~K}$ and there is a clear trend of increasing temperature at increasing distance to the stars.

The radial velocity of $\mathrm{H}_{2}$ emitting gas has been calculated from line fitting to the $v=1-0 S(1)$ line. The radial velocities in the vicinity of $\mathrm{T}$ Tau $\mathrm{N}$ are very close to the rest velocity of the 
star and are associated with a nearly face-on circumstellar disk (Gustafsson et al. 2008). The shocks southeast and southwest of T Tau S are $\sim 20 \mathrm{~km} \mathrm{~s}^{-1}$ blueshifted with respect to the stars, while the shocks northwest of T Tau $\mathrm{S}$ are $\sim 7 \mathrm{~km} \mathrm{~s}^{-1}$ blueshifted. All the shocked gas surrounding T Tau $\mathrm{S}$ is thus blueshifted.

Based on data taken two years prior to our observations (Herbst et al. 2007) we have measured the proper motions of the observed shocks. These show that the southwest flow is moving away from $\mathrm{T}$ Tau $\mathrm{Sb}$ with a speed of $36 \mathrm{~km} \mathrm{~s}^{-1}$ at an inclination of $\sim 60^{\circ}$. The southeast flow is moving at a higher speed of $57 \mathrm{~km} \mathrm{~s}^{-1}$ at an inclination of $\sim 70^{\circ}$. In contrast, there are no detectable proper motions for the shocks northwest and west of $\mathrm{T}$ Tau S, which suggests that they move at lower velocities or close to the line of sight.

We have modelled seven individual flows in $\mathrm{T}$ Tau with state of the art $\mathrm{C}$-shock models. The modelling shows that the ambient medium in which the shocks propagate is very dense with preshock densities of $5 \times 10^{5} \mathrm{~cm}^{-3}$. All flows are consistent with being excited by a shock wave at a speed of $17-24 \mathrm{~km} \mathrm{~s}^{-1}$ in a $B=0.35-0.70 \mathrm{mG}$ medium. The inferred shock velocity is lower than the measured $3 \mathrm{D}$ velocity, which might suggest that the gas around $\mathrm{T}$ Tau has been accelerated by an earlier passing shock wave.

We detect strong [Fe II] emission northwest and south of $\mathrm{T}$ Tau $\mathrm{S}$. The $\left[\mathrm{Fe}\right.$ II] and $\mathrm{H}_{2}$ emission are not spatially coincident. [Fe II] is mainly produced in J-type shocks where $\mathrm{H}_{2}$ is dissociated. There is a strong overlap between the distribution of [Fe II] emission and UV flourescent $\mathrm{H}_{2}$ emission. The UV flourescence might be powered by $J$-shocks.

Based on the SINFONI data together with the proper motions we propose that outflows from all three stars in the T Tau system contribute to the $\mathrm{H}_{2}$ emission in the immediate vicinity of T Tau S. T Tau N drives the shocks northwest of T Tau S and is associated with the east-west outflow. T Tau Sa drives the flows southeast of the southern binary and is the source of the southeast-northwest outflow. T Tau Sb is the origin of a previously undetected outflow in the southwest direction. The proposed association of stars and outflows implies that all three disks are misaligned.

Acknowledgements. Part of this paper was written during an extended visit to Aarhus University, Denmark. M.G. gratefully acknowledge the support of Aarhus University and MPIA in order to make this possible.

\section{References}

Akeson, R. L., Koerner, D. W., \& Jensen, E. L. N. 1998, ApJ, 505, 358 Beck, T. L., Prato, L., \& Simon, M. 2001, ApJ, 551, 1031

Beck, T. L., Schaefer, G. H., Simon, M., et al. 2004, ApJ, 614, 235 Beck, T. L., McGregor, P. J., Takami, M., et al. 2008, ApJ, 676, 472 Bevington, P. 1969, Data reduction and Error Analysis for the Physical Sciences (New York: McGraw-Hill)

Black, J. H., \& van Dishoeck, E. F. 1987, ApJ, 322, 412

Böhm, K., \& Solf, J. 1994, ApJ, 430, 277

Bührke, T., Brugel, E. W., \& Mundt, R. 1986, A\&A, 163, 83

Campins, H., Rieke, G. H., \& Lebofsky, M. J. 1985, AJ, 90, 896

Duchêne, G., Ghez, A. M., \& McCabe, C. 2002, ApJ, 568, 771

Duchêne, G., Ghez, A. M., McCabe, C., et al. 2005, ApJ, 628, 832

Duchêne, G., Beust, H., Adjali, F., Konopacky, Q. M., \& Ghez, A. M. 2006, A\&A, 457, L9

Dyck, H. M., Simon, T., \& Zuckerman, B. 1982, ApJ, 255, L103

Eisenhauer, F., Abuter, R., Bickert, K., et al. 2003, in Instrument Design and Performance for Optical/Infrared Ground-based Telescopes, ed. M. Iye, $\&$ A. F. M. Moorwood, Presented at the Society of Photo-Optical Instrumentation Engineers (SPIE) Conf., Proc. SPIE, 4841, 1548

Eislöffel, J., \& Mundt, R. 1998, AJ, 115, 1554

Flower, D. R., \& Pineau des Forêts, G. 2003, MNRAS, 343, 390

Flower, D. R., Le Bourlot, J., Pineau des Forêts, G., et al. 2003, MNRAS, 341, 70

Gustafsson, M., Labadie, L., Herbst, T. M., et al. 2008, A\&A, 488, 235

Gustafsson, M., Ravkilde, T., Kristensen, L., et al. 2010, A\&A, A\&A, 513, A5

Hartmann, L., Hewett, R., Stahler, S., et al. 1986, ApJ, 309, 275

Herbst, T. M., Beckwith, S. V. W., Glindemann, A., et al. 1996, AJ, 111, 2403

Herbst, T. M., Robberto, M., \& Beckwith, S. V. W. 1997, AJ, 114, 744

Herbst, T. M., Hartung, M., Kasper, M. E., Leinert, C., \& Ratzka, T. 2007, AJ, 134, 359

Joy, A. H. 1945, ApJ, 102, 168

Köhler, R. 2008, J. Phys. Conf. Ser., 131, 012028

Koresko, C. D. 2000, ApJ, 531, L147

Kristensen, L. E., Ravkilde, T. L., Field, D., Lemaire, J. L., \& Pineau Des Forêts, G. 2007, A\&A, 469, 561

Loinard, L., Rodríguez, L. F., D’Alessio, P., Rodríguez, M. I., \& González, R. F. 2007a, ApJ, 657, 916

Loinard, L., Torres, R. M., Mioduszewski, A. J., et al. 2007b, ApJ, 671, 546

Mathis, J. S. 1990, ARA\&A, 28, 37

Ratzka, T., Schegerer, A. A., Leinert, C., et al. 2009, A\&A, 502, 623

Ray, T. P., Muxlow, T. W. B., Axon, D. J., et al. 1997, Nature, 385, 415

Robberto, M., Clampin, M., Ligori, S., et al. 1995, A\&A, 296, 431

Saucedo, J., Calvet, N., Hartmann, L., et al. 2003, ApJ, 591, 275

Solf, J., \& Böhm, K.-H. 1999, ApJ, 523, 709

Turner, J., Kirby-Docken, K., \& Dalgarno, A. 1977, ApJS, 35, 281

van Boekel, R., Güdel, M., Henning, T., et al. 2009, A\&A, 497, 137

van den Ancker, M. E., Wesselius, P. R., Tielens, A. G. G. M., van Dishoeck,

E. F., \& Spinoglio, L. 1999, A\&A, 348, 877

Walter, F. M., Herczeg, G., Brown, A., et al. 2003, AJ, 126, 3076 